\title{
1 Genomic signatures of host-specific selection in a parasitic
} 2 plant

Authors: Emily S. Bellis ${ }^{1,2,3}$, Clara S. von Münchow ${ }^{4}$, Calvins O. Odero ${ }^{5}$, Alan Kronberger ${ }^{1}$, Elizabeth Kelly ${ }^{3}$, Tian $\mathrm{Xia}^{3}$, Xiuzhen Huang ${ }^{1,2}$, Susann Wicke ${ }^{4,5}$, Steven M. Runo ${ }^{6}$, Claude W. dePamphilis ${ }^{3}$, Jesse R. Lasky ${ }^{3}$

\section{Target journal: AJB}

Corresponding Author: Emily S. Bellis, ebellis@astate.edu

${ }^{1}$ Department of Computer Science, Arkansas State University, State University, AR, USA

${ }^{2}$ Center for No-Boundary Thinking, Arkansas State University, State University, AR, USA

${ }^{3}$ Department of Biology, Pennsylvania State University, University Park, PA, USA

${ }^{4}$ Institute for Biology, Humboldt University of Berlin, Berlin, Germany

${ }^{5}$ Späth-Arboretum of the Humboldt University of Berlin, Berlin, Germany

${ }^{6}$ Department of Biochemistry, Microbiology, and Biotechnology, Kenyatta University, Nairobi, Kenya

Manuscript received: ; revision accepted:

Running head: Striga hermonthica population genomics

\section{ABSTRACT}

Premise-Parasitic plants and their hosts are emerging model systems for studying genetic variation in species interactions across environments. The parasitic plant Striga hermonthica (witchweed) attacks a range of cereal crop hosts in Africa. Striga hermonthica exhibits substantial genetic variation in host preference and in specificity versus generalism.

Some of this variation is locally adapted, but the genetic basis of specialization on certain hosts is unknown.

Methods-We present an alignment-free analysis of population diversity in $S$. hermonthica using whole genome sequencing (WGS) data for 68 individuals from western Kenya. We validate our reference-free approach with germination experiments and a de novo assembled draft genome. 
Results-K-mer based analyses reveal high genome-wide diversity within a single field, similar to values between individuals collected $100 \mathrm{~km}$ apart or farther. Analysis of hostassociated $k$-mers implicated genes involved in development of the parasite haustorium (a specialized structure used to establish vascular connections with host roots) and a potential role of chemocyanins in molecular host-parasitic plant interactions. Conversely, no phenotypic or genomic evidence was observed suggesting host-specific selection on parasite response to strigolactones, hormones exuded by host roots and required for parasite germination.

Conclusions-This study demonstrates the utility of WGS for plant species with large, complex genomes and no available reference. Contrasting with theory emphasizing the role of

43 early recognition loci for genotype specificity, our findings support host-specific selection on

44 later interaction stages, suggesting recurring host-specific selection each generation alternating

45 with homogenizing gene flow.

47 Key words: population genomics, recurrent selective sweeps, agroecosystems,

48 Orobanchaceae, host-parasite coevolution, chemocyanin

\section{INTRODUCTION}

Characterizing the genomic basis of adaptation to local biotic environments is a key

51 challenge for evolutionary ecology (Ebert and Fields, 2020). Parasites and mutualists exert

52 strong influences on host fitness, and may even constitute the predominant selective pressure

53 shaping patterns of local adaptation in some systems (Fumagalli et al., 2011; Castellano et al.,

54 2019). Compared to abiotic environmental gradients, adaptation to biotic environments may be

55 characterized more frequently by selection on fewer mutations of large effect, due to selection

56 on a more rapidly changing adaptive landscape (Wilfert and Schmid-Hempel, 2008; Louthan

57 and Kay, 2011). However, many host-parasite and host-mutualist systems involve a complex 
58 multi-step infection process including many stages of interaction between host and symbiont

59 derived molecules (Hall et al., 2017).

An outstanding question is whether adaptation to local biotic environments occurs most

61 often via selection on genes involved during initial infection stages, or whether genetic variation

62 at later stages of the interaction is also frequently maintained. The expectation from theoretical

63 studies is that initial recognition loci are more likely than downstream effector loci to contribute

64 to host genotype by parasite genotype interactions $\left(G_{H} \times G_{P}\right)$ and correspondingly, local

65 adaptation (Nuismer and Dybdahl, 2016). The first prediction, that recognition loci contribute

66 more to $G_{H} \times G_{P}$, is supported by empirical studies of the waterflea Daphnia magna and its

67 bacterial parasite Pasteuria ramosa (Hall et al., 2017). In this system, most of the genetic

68 variance in parasite infection was associated with a single major effect QTL linked to the early

69 stage of parasite attachment (Hall et al., 2019). In contrast, many different QTLs of smaller

70 effect were associated with later stages, highlighting the potential for independent evolution of

71 traits involved in different stages of the infection process (Hall et al., 2019). Supporting the

72 second prediction that selection on recognition traits often underlies local adaptation (Nuismer

73 and Dybdahl, 2016), studies of plant pathosystems have revealed reciprocal coevolutionary

74 selection on host resistance $(R)$ genes and parasite avirulence genes, for example in the flax-

75 flax rust system (Ravensdale et al., 2011; Thrall et al., 2012). However, a high degree of

76 genotype specificity has also been observed for many host-parasite systems characterized by

77 more quantitative mechanisms of resistance (Poland et al., 2009). For host-parasite interactions

78 characterized by quantitative genetic architectures, we still know little regarding the genetic

79 basis of local adaptation in natural populations and the extent to which initial vs. later infection

80 stages contribute to $G_{H} \times G_{p}$.

81 An emerging model system for studying spatial pattern and process in coevolutionary

82 genomics is the parasitic plant Striga hermonthica and its cereal hosts. In contrast to Striga

83 gesneriodes, which parasitizes cowpea via a qualitative gene-for-gene mechanism, host 
84 resistance to S. hermonthica is highly polygenic (Li and Timko, 2009; Timko et al., 2012) with at 85 least one large effect locus (Gobena et al., 2017). Striga hermonthica parasitizes grass hosts 86 including sorghum, maize, rice, and millets and is one of the greatest biotic constraints to food 87 security in Africa (Ejeta, 2007; Spallek et al., 2013; Savary et al., 2019). An individual S.

88 hermonthica plant can produce thousands of seeds that may survive in the soil for a decade or 89 more under optimal conditions (Bebawi et al. 1984; but see Gbèhounou et al. 2003).

Parasite seeds germinate by detecting strigolactones (SLs), hormones exuded from host

91 roots under nutrient-deficient conditions that also stimulate host interactions with beneficial

92 mycorrhizal fungi (Akiyama et al., 2005). Parasite perception of SLs is mediated through binding

93 to paralogs of KARRIKIN INSENSITIVE 2 (KAI2), known as HYPOSENSITIVE TO LIGHT (HTL)

94 proteins. SL receptors of the $K A I 2 d$ clade rapidly expanded and diversified during the transition

95 to parasitism in the Orobanchaceae, a plant family that includes thousands of mostly parasitic

96 species (Conn et al., 2015). For example, the $\sim 600 \mathrm{Mb}$ genome of Striga asiatica contains 21

97 KAl2 genes, many of which occur as tandem duplications (Yoshida et al., 2019). Striga

98 hermonthica may contain 13 or more KAl2 paralogs (Nelson, 2021), including 11 for which the

99 binding affinity for diverse SLs has been extensively characterized (Toh et al., 2015; Tsuchiya et

100 al., 2015). The continent-wide distribution of host-specific S. hermonthica populations suggests

101 adaptation to their local host communities, via generalization where diverse hosts are available

102 and specialization where a particular host is common (Bellis et al., 2021). Given the low

103 germination of millet- and sorghum-specific S. hermonthica populations in response to root

104 exudates from the alternate host (Parker and Reid, 1979), it is possible that at least some of this

105 host-specificity results from natural selection on SL perception. Host-specific germination of

106 Striga species could result from expanded SL response by different KAI2 paralogs, or

107 evolutionary fine-tuning of SL perception if some SL receptors instead function to inhibit

108 germination (Nelson, 2021). 
Following SL perception and parasite germination, host-derived phenolic compounds

110 induce formation of the haustorium, the specialized multicellular feeding structure used by

111 parasitic plants to invade host tissues (Cui et al., 2018). Intrusive cells of the haustoria invade

112 host tissues to form direct connections with host vasculature (Masumoto et al., 2021). Water,

113 nutrients, and other molecules including mRNA (Kim et al., 2014), small RNA (Shahid et al.,

114 2018), DNA (Yang et al., 2019), and proteins (Liu et al., 2020; Shen et al., 2020) are directly

115 transferred through haustorial connection. In addition to natural variation in low germination

116 stimulation (Dayou et al., 2021; Mallu et al., 2021), post-germination host resistance in Striga

117 hermonthica is often apparent across diverse hosts, for example due to induction of an intense

118 hypersensitive response, formation of a mechanical barrier, or failure of the parasite to form

119 vascular connections (Mbuvi et al., 2017; Mutinda et al., 2018; Kavuluko et al., 2020). Much of

120 this genetic variation in natural resistance may result from host populations' local adaptation to

121 parasitism across the range of Striga hermonthica (Bellis et al., 2020).

Extensive genetic and germplasm resources have enabled broad-scale studies of local

123 adaptation in Striga hosts (Bellis et al., 2020), but understanding reciprocal adaptation in

124 parasite populations is challenged by paucity of genomic data for S. hermonthica. At the

125 population-scale, restriction-site associated DNA sequencing (RAD-Seq) and analysis of

126 polymorphism in transcriptomes have begun to shed light on population level diversity in these

127 parasites (Unachukwu et al. 2017; Lopez et al. 2019). However, reduced representation

128 approaches pose difficulties if only a fraction of host genome diversity is tagged by RAD-Seq

129 markers (Lowry et al., 2017) or (in the case of transcriptomes) if genes under selection are not

130 expressed in sequenced tissues. Like other parasitic angiosperms, S. hermonthica is

131 characterized by a larger genome than non-parasitic relatives (Lyko and Wicke, 2021), with

132 estimated size of $\sim 1 \mathrm{~Gb}(1 \mathrm{C}=0.9 \mathrm{~Gb}$; Yoshida et al. 2010) or greater (1C = $1.4 \mathrm{~Gb}$; Estep et al.

133 2012). Striga hermonthica is a highly heterozygous obligate outcrosser, posing additional

134 challenges for genome assembly and reference-based approaches. Here, we provide a 
reference-free analysis of population-scale diversity in $S$. hermonthica, based on whole genome sequencing (WGS) data. Using a unique alignment-free bioinformatic approach, we identify

137 genetic variation associated with host-specific parasitism in natural populations and investigate 138 signatures of selection surrounding these loci. Based on these findings, we evaluate the 139 hypothesis that adaptation to local host populations results from selection on genes involved in 140 early stages of the interaction (SL perception) against the alternative that selection on genes 141 involved in later stages is primarily responsible.

\section{MATERIALS AND METHODS}

145 individuals in July 2018 from six locations in western Kenya (Table S1). Two plots with $S$. 146 hermonthica parasitizing different host species were sampled at each location, chosen as close 147 as possible and in most cases, from nearby plots on the same farm (i.e. within 15 meters). Per 148 plot, twelve S. hermonthica individuals were chosen haphazardly, with effort taken to sample 149 individuals distributed evenly throughout the plot. This sampling design was expected to result 150 in the selection of individuals from the same interbreeding population that are relatively

151 homogeneous across their genetic background due to high rates of gene flow among

152 neighboring plots. However, sampling mature plants could allow for identification of loci under

153 selection for parasitism on a specific host due to a single generation of selection. Individuals

154 were photographed before collection and images for representative individuals at each site 155 uploaded to iNaturalist. Three to four leaves per individual were sampled directly into silica gel, 156 before collection of the whole individual into separate paper bags. Plants were dried in paper

157 bags before harvesting and manual cleaning of seeds. Cleaned seeds were shipped to Penn

158 State for germination rate experiments and stored in individual $2 \mathrm{~mL}$ microcentrifuge tubes at 159 room temperature prior to experiments. Five voucher specimens (ESB collection numbers 1602018.1 to 2018.5) were deposited in the collection of the East African Herbarium (EA). 
Whole genome sequencing-For samples collected in 2018, whole genome sequencing was performed for a subset of 68 S. hermonthica individuals. This included all 24

164 individuals collected from adjacent plots of finger millet and maize in Kisii, all 24 individuals 165 collected from adjacent plots of sorghum and maize in Homa Bay, and five individuals (two or

166 three per plot) from four additional locations. DNA was extracted from silica-dried leaf tissue in 167 the USDA APHIS quarantine facility at the Pennsylvania State University using the E.Z.N.A.

168 Plant DNA DS Mini Kit (Omega Bio-tek, Norcross, Georgia, USA) according to the

169 manufacturer's protocol. Genomic library preparation and paired-end 150 bp sequencing was 170 carried out by the Texas A\&M AgriLife Genomics and Bioinformatics Service, on a single lane of 171 a NovaSeq 6000 S4 flow cell.

Population structure - We followed a reference-free approach to evaluate population

174 genomic patterns among sequenced samples. Raw reads that could be classified as plant-

175 derived were identified using Kraken 2 (Wood et al., 2019), based on a custom database built

176 from the complete set of plant genomes and proteins in the NCBI RefSeq collection, sequences

177 from $472 \mathrm{Mbp}$ of the Striga asiatica genome (Yoshida et al., 2019) and S. hermonthica

178 transcriptome sequences (build StHeBC4) from the Parasitic Plant Genome Project II (Yang et

179 al., 2014). Classified sequences were trimmed using BBduk from BBTools (Bushnell, n.d.),

180 removing sequence on the ends of reads with low quality (qtrim=rl trimq=20 minlen=50) or 3'

181 matches to adapters $(\mathrm{k}=23$ mink=11 hdist=1 tpe tbo $\mathrm{ktrim}=\mathrm{r})$. To reduce bias associated with

182 differences in per-sample read depth, reads were downsampled to 5.2 Gbp with BBTools

183 Reformat.

Mash, a dimensionality reduction technique based on the MinHash algorithm, was used

185 to estimate genetic distance between samples based on resulting read sets (Ondov et al., 186 2016). Mash previously showed improved performance compared to alignment-based methods 
187 for estimating pairwise genetic distance for polyploid plant genomes using simulated and real

188 data (VanWallendael and Alvarez, 2020). We used a k-mer size of 31, removing $k$-mers with

189 less than 2 copies but increasing the sketch size to $1 \times 10^{7}$ to account for a larger volume of

190 input data. Principal Coordinates Analysis was performed in R version 4.0 with the pcoa function

191 of the 'ape' package (Paradis and Schliep, 2019). A smaller $k$-mer size $(k=21)$ was also tested

192 but did not alter clustering patterns in PCoA. Correlation between the genetic distance matrix

193 and the geographic distance matrices, calculated with 'geodist', was determined using a Mantel

194 test (Padgham and Sumner, 2021).

Germination experiments-Seed germination was assayed in the USDA-APHIS-

197 permitted quarantine lab at Pennsylvania State University (permit no. P526P-21-04540). A

198 detailed step-by-step protocol is available from protocols.io repository (Bellis and Kelly, 2019),

199 following the modifications for testing seeds collected from individual plants. Briefly, seeds were

200 surface sterilized for 10 minutes in $1.5 \mathrm{~mL}$ microcentrifuge tubes with a $0.5 \%$ sodium

201 hypochlorite solution before preconditioning for 12 days at $30^{\circ} \mathrm{C}$ in separate wells of foil-

202 wrapped 12-well culture plates, with three technical replicates per unique germination stimulant

203 and parasite genotype combination. Germination counts were performed 3 days after addition of

204 germination stimulants. Tested germination stimulants included (+)5-deoxystrigol (Olchemim,

205 Olomouc, Czech Republic; CAS: 151716-18-6) or ( \pm )orobanchol (Olchemim; CAS: 220493-64-1)

206 at $0.01 \mu \mathrm{M}$ and ( \pm )-GR24 (Chempep, Wellington, Florida, USA; CAS: 76974-79-3) at $0.2 \mu \mathrm{M}$.

207 GR24 is a synthetic strigolactone analog commonly used in laboratory germination studies of

208 parasitic plants as a positive control. 5-deoxystrigol is one of the major SLs produced by

209 compatible grass hosts (Awad et al., 2006) and is a potent stimulator of parasite germination

210 whereas orobanchol is a more dominant SL among dicot hosts (Yoneyama et al., 2008) and is a

211 less potent stimulator of $S$. hermonthica germination for certain genotypes (Cardoso et al.,

212 2014). Specifically, S. hermonthica tested populations from Mali and Niger germinate poorly in 
213 response to orobanchol (Haussmann et al., 2004; Bellis et al., 2020) whereas Kenyan S.

214 hermonthica show a greater ability to germinate in response to exudate from sorghum hosts

215 carrying loss-of-function mutations at the sorghum resistance locus LOW GERMINATION

216 STIMULANT 1 (LGS1), which results in high amounts of orobanchol rather than 5-deoxystrigol

217 in root exudates (Gobena et al., 2017).

218 In addition to seed collections kept separately from individual plants, we also included

219 tests of bulk seed collected from the Kibos population that were confirmed to have high

220 germinability in our previous experiments (Bellis et al., 2020). We used a generalized linear

221 mixed model (GLMM) with a random effect of $S$. hermonthica genotype (of the parent plant) to

222 compare differences in germination rate among sites, hosts, and treatments (orobanchol vs. 5-

223 deoxystrigol). GLMMs were implemented in R version 4.0 with the Ime4 package (Bates et al.

224 2015).

Host-specific differentiation-We next sought to identify particular genomic regions

227 differentiated between parasites growing on different hosts. This analysis targeted parasite

228 populations from Homa Bay or Kisii, for which we sequenced DNA from parasites for two

229 different hosts on the same farm from immediately adjacent plots. For the Kisii population, the

230 dataset included individuals from finger millet and maize, whereas for the Homa Bay population

231 the dataset included parasites from sorghum and maize ( $n=12$ from each host species; 48

232 individuals total). Counts for $k$-mers of length 31 were summarized across sequenced

233 individuals using HAWK (Rahman et al., 2018) and used to calculate the fixation index, $G_{S T}$ (Nei

234 and Chesser, 1983), for each $k$-mer using custom Python scripts. $G_{S T}$ is a generalization of the

235 widely used fixation index $F_{S T}$ applicable to non-diploid loci (Nei, 1973). To extend $G_{S T}$ to our

236 reference-free genotyping approach, at each $k$-mer we considered two allelic states (present or

237 absent) where the $k$-mer was marked as present in an individual if it was counted at least once

238 or absent if it was not observed at all. 
To gather functional information for host-associated $k$-mers, 31 -mers with $G_{S T}$ above 0.5 were extracted and assembled into longer contigs using ABYSS 2.0.2, specifying a $k$-mer length

241 of 29 for assembly (Jackman et al., 2017). Assembled contigs were then queried against contigs

242 from two published S. hermonthica transcriptome assemblies using BLAST optimized for short

243 sequences (blastn-short). Transcriptome assemblies in our BLAST database included StHeBC4

244 (265,694 scaffolds covering 369.7 Mb) from the Parasitic Plant Genome Project (Westwood et

245 al., 2012) and Sh14v2 (81,559 scaffolds covering 83.9 Mb) from Yoshida et al. (2019).

246 Annotations are based on the top hit from the Sh14v2 transcriptome.

247 Contigs assembled from host-associated $k$-mers were further studied by mapping

248 cleaned sample reads to mRNA reference sequences, following the strategy from (Therkildsen

249 and Palumbi, 2017). Mapping to a transcriptome reference has the potential to introduce errors

250 in SNP calling due to intron/exon boundaries and the short length of transcripts, so we restricted

251 this analysis to a small set of loci for which alignments could be manually inspected. Sequences

252 in our reference included three transcripts with potential functions in haustorium development

253 (StHeBC4_h_c11261_g0_i1, StHeBC4_p_c12587_g2_i1, StHeBC4_u_c12903_g27039_i4; see

254 Results). The reference also included transcript sequences for a set of 11 previously

255 characterized S. hermonthica strigolactone receptors [GenBank accession numbers KR013121

256 - KR13131] (Tsuchiya et al., 2015). High quality, contaminant-filtered reads were mapped to the

257 reference transcriptome using BWA-MEM ( $\mathrm{Li}, 2013)$, and alignments with low quality were

258 removed using samtools view (-q 20) (Li et al., 2009). Allele frequencies for each site in the

259 reference transcriptome were estimated based on genotype likelihoods using ANGSD, ignoring

260 low quality bases (-minQ 25) and allowing reads for which only one end mapped (-

261 only_proper_pairs 0) (Kim et al., 2011; Korneliussen et al., 2014). Sites with information for

262 fewer than nine of twelve individuals in the population were excluded, and the difference in

263 estimated allele frequency between parasite populations on different hosts was visualized with

$264 \mathrm{R}$ version 4.0 (R Core Team, 2020). This strategy was used to filter false SNP calls due to 
errors in mapping DNA-derived reads to a transcriptome reference, since these SNPs should be observed as a fixed difference from the reference that occurs in both populations. We further investigated genes with potential structural variation by extracting reads aligned to the transcript reference and their unmapped pairs and reassembling them with ABYSS ( $k=51)$ (Jackman et al., 2017).

Validation of k-mer-based approaches-To investigate patterns of selection surrounding putative host-associated loci and validate findings from reference-free analyses, we also mapped reads generated for $S$. hermonthica to a draft reference assembled specimen,

274 grown ex situ from seeds collected on maize in the Irimbi district of Southern Uganda

275 (specimens voucher deposited at MSUN). DNA was extracting from developing leaves and 276 inflorescences of one individual using a modified 1x CTAB-protocol with subsequent PEG-8000 277 precipitation and purification (Wicke et al., 2016). Genomic libraries were sequenced on an 278 Illumina HiSeq 2000 in 101 bp paired-end mode at Eurofins GATC Biotech GmbH (Constance, 279 Germany). Additional data were generated to a targeted depth of 110X using the HiSeq 2500 280 platform using the HiSeq SBS Kit v4 at Eurofins GATC Biotech GmbH, for which DNA from the 281 original extract was subjected to $\$ 29-$ polymerase based whole-genome amplification. Whole 282 genome-amplified DNA was size-selected for $>20 \mathrm{~kb}$ fragments on $1 \%$ low-melting point 283 agarose and purified using an agarose digest-based purification from gel with subsequent 284 ethanol/sodium acetate precipitation (Wicke et al., 2013). For the final assembly, we employed 285 Trimmomatic v0.36 (Bolger et al., 2014) to remove adapters and retain only sequences longer 286 than 36 bp with an average per-base quality above 15 ("ILLUMINACLIP:TruSeq3-PE.fa":

287 2:30:10 SLIDINGWINDOW:4:15 MINLEN:36). The quality-trimmed data were assembled using 288 SPAdes v3.10.1 with $k$-mer sizes of 21, 33, 55, and 77 (Bankevich et al., 2012). Assembly 289 quality was ascertained using Quast v4.5 (Gurevich et al., 2013) with default parameters for 290 eukaryotes. The resulting assembled contigs were contaminant-filtered, for which we ran a 
nucleotide BLAST search of all contigs against the non-redundant nucleotide database (access date: 10.07.2017) using BLAST+ v2.6 with an e-value of $1 \mathrm{E} 10^{-4}$. Only contigs with the three best hits matching to green land plants (Viridiplantae) were retained.

After removing scaffolds shorter than $500 \mathrm{bp}$ from the reference, high quality sequences from each S. hermonthica individual were mapped using BWA-MEM v0.7.17 (Li, 2013). Sequences mapping with quality less than 20 were excluded using SAMtools v1.10 (Li et al., 297 2009). Tajima's D was calculated in non-overlapping windows of 1 -kb using ANGSD v0.935 298 based on the folded site frequency spectrum and including reads where only one end mapped (299 only_proper_pairs 0), to account for the highly fragmented nature of the assembly (Korneliussen 300 et al., 2014). $F_{S T}$ was also calculated in non-overlapping windows with ANGSD, using the 301 SAMtools method for calculating genotype likelihoods. Genome-wide mean values of Tajima's D were determined by fitting an intercept-only linear mixed model to window estimates of $F_{S T}$ or

303 Tajima's D, including a random effect of 'contig' to account for increased correlation among measurements from nearby genomic regions, with the R package Ime4 (Bates et al., 2015). An empirical $P$-value for Tajima's D for the contig containing the chemocyanin was calculated based on the number of 10,000 randomly sampled windows of size matching the assembled

307 length of the contig $(1-\mathrm{kb})$ with values more extreme than the observed value.

To validate the presence/absence polymorphism for the chemocyanin gene, we performed PCR using primers designed from the reassembled 'finger millet' allele (Primer Set A: 5'-AAGATTGCGGTTACCACCAG-3' and 5'-TCTCGATCCTTTTGGAATGG-3') and the

311 transcript reference (Primer Set B: 5'- CAGGAGCAAGTAGAGTAGAGCA-3' and 5'-

312 TGGGGAAAGAGGTAGTGCAA-3'). PCR was performed with DreamTaq DNA Polymerase 2x

313 Mastermix (ThermoFisher, Waltham, Massachusetts, USA) under the following cycling

314 conditions: $95^{\circ} \mathrm{C}$ for $3 \mathrm{~min} ; 30$ cycles of $95^{\circ} \mathrm{C}(30 \mathrm{~s}), 50.3^{\circ} \mathrm{C}(30 \mathrm{~s}), 72^{\circ} \mathrm{C}(60 \mathrm{~s}) ; 72^{\circ} \mathrm{C}$ for $5 \mathrm{~min}$. 
Sequencing-For the 68 Kenyan samples, on average $80 \%$ of reads per library were classified as plant-derived using Kraken 2 (range: $71-86 \%$ ). The majority of classified reads matched the $S$. hermonthica transcriptome (mean: 46\%) or the S. asiatica genome (mean:

$32030 \%)$. After quality and adapter trimming, on average 7.7 Gigabase pairs of plant-derived 321 sequence data remained per sample (range: 5.2-11.7 Gbp). Given flow cytometry estimates of 322 the genome size of $S$. hermonthica ranging from $1 \mathrm{C}=0.9 \mathrm{~Gb}$ (Yoshida et al., 2010) to $1 \mathrm{C}=1.4$ $323 \mathrm{~Gb}$ (Estep et al., 2012), this sequencing effort corresponds to an approximate average depth of $3245.5 x$ to $8.6 x$ read coverage per base for each sample.

Population structure - In contrast to previous studies based on microsatellite markers (Gethi et al., 2005) but consistent with results from GBS (Unachukwu et al., 2017), our analyses suggest that geography is a primary factor shaping population structure in $S$. hermonthica from western Kenya. Principal Coordinates Analysis (PCoA) based on k-mers indicated high genetic diversity within populations, with only subtle differentiation of populations from the same farm collected from different hosts (Fig. 1). Greater correlation was observed between genetic and geographic distance than expected by chance $(p=0.001$, Mantel test), and patterns of genetic variation were consistent with those expected under isolation by distance (Fig. 1F). Although

334 populations were structured by distance, within-population diversity was very high at the geographic scale investigated. Many individuals exhibited a comparable range of pairwise genetic diversity within a single field $(k$-mer distance ranging from $0.0216-0.0286 ; n=572$ pairwise comparisons) as between individuals sampled more than $100 \mathrm{~km}$ apart ( $k$-mer distance ranging from $0.0242-0.0286 ; n=336$ pairwise comparisons; Fig. 1F).

341 locations in Africa, S. hermonthica populations from western Kenya demonstrate a generalist 342 germination response to strigolactones (Haussmann et al., 2004; Bellis et al., 2020). However, 
Striga germination tests are typically conducted with bulk seeds collected from many individuals

344 in a field, potentially masking individual-level variation that could be segregating with respect to

345 parasitism on different hosts, for example if a generalist population is composed of individuals

346 that specialize on different resources (Bolnick et al., 2002).

To characterize individual-level variation in western Kenyan S. hermonthica, we

348 conducted controlled germination tests in the USDA-permitted quarantine facility at

349 Pennsylvania State University. Positive controls with bulk seed (collected in the Kibos region)

350 and $0.2 \mu \mathrm{M}$ of the artificial strigolactone GR24 indicated good germinability for positive controls

351 (66.5\%), and no germinated seeds were observed in wells with only sterile water. Compared to

352 the higher concentration of GR24, bulk seed showed slightly lower germination rates in

353 response to $0.01 \mu \mathrm{M}$ of the natural SLs orobanchol (51.0\%) and 5-deoxystrigol (55.7\%)

354 indicating that the concentrations of orobanchol and 5-deoxystrigol used in the individual-level

experiment should produce strong but sub-maximal germination responses.

We did not find significant host-associated germination variation among seeds collected

357 from individual parasites on different hosts (Table S1; Fig. 2). After accounting for treatment and

358 site, host-of-origin was not a statistically significant effect in our model $(P=0.247$; likelihood

359 ratio test for test of full vs. reduced GLMM). Comparing individuals from Kisii to Homa Bay, the

360 probability of germination was 30\% lower $(P=0.04$; GLMM with fixed effects of 'Site' and

361 'Treatment') and 15\% lower in response to orobanchol than to 5-deoxystrigol $(P=0.004$;

362 GLMM).

Host-associated loci-Germination tests did not support host-specific differences in

365 strigolactone response in these populations. However, it is possible that host-specific selection

366 could still leave detectable signatures at the genetic level. We identified host-associated genetic

367 variation without a reference using a $k$-mer based approach. Highly differentiated $k$-mers were

368 defined as those having $G_{S T}>0.5$ (Fig. S2). A greater proportion of 31-mers were highly 
differentiated for parasites on finger millet vs. maize hosts in Kisii (4.8\% of 31-mers) compared to sorghum vs. maize hosts in Homa Bay (0.6\% of 31-mers; Fig. S1).

Highly differentiated 31 -mers were then assembled into longer contigs for follow-up

372 analysis. For the Homa Bay population (maize vs. sorghum hosts), 42 contigs were assembled

373 ranging in length from 58 to $91 \mathrm{bp}$. Sixteen of these contigs had good BLAST hits to the

374 transcriptome database, with $>85 \%$ identity over at least 45 bp (Table 1). For the Kisii

375 population (finger millet vs. maize hosts), highly differentiated $k$-mers assembled into 469

376 contigs with length ranging from 29 to $211 \mathrm{bp}$. Of these, 241 had good BLAST hits to transcripts

377 in our database (Table S2).

378 Of particular interest to our investigation were host-specific contigs with high levels of

379 similarity to known Striga parasitism genes including SL receptors and genes involved in

380 haustorium development. We first searched for similarity to a set of $11 \mathrm{~S}$. hermonthica

381 strigolactone receptors that vary in their binding affinity for diverse strigolactones [GenBank

382 accession numbers KR013121 - KR13131] (Tsuchiya et al., 2015). We observed only one likely

383 spurious match to ShHTL2 (87\% similarity over $23 \mathrm{bp})$ and no hits to any of the $21 \mathrm{KAl}$

384 paralogs from the Striga asiatica genome (Yoshida 2019). In follow-up analyses based on

385 alignments to reference ShHTL transcripts, only one site in ShHTL6 had an estimated difference

386 in allele frequency between parasites on finger millet and maize exceeding 0.5 (Fig. S2). This

387 polymorphism occurred at position 457 and does not result in an amino acid change. Together,

388 we find little phenotypic or genomic evidence supporting host-specific differentiation for loci

389 involved in perception of strigolactones in these populations.

390 In contrast, several assembled host-specific contigs had good matches to loci implicated

391 in development of haustoria (Fig. 3). Annotated transcripts included a 60 bp contig with $>98 \%$

392 similarity over its full length to a transcript annotated as SUPPRESSOR OF G2 ALLELE SKP1

393 (SGT1) (Table S1). SGT1 was among the top upregulated genes in haustoria of the root

394 parasitic plant Thesium chinense and was hypothesized to be important for generating auxin 
response maxima during haustorium development (Ichihashi et al., 2017). In S. hermonthica, it is also highly expressed in imbibed seeds (Stage 0 ) and in haustoria attached to host roots

397 (Stage 3; Fig. 3D). Parasitism on different hosts was associated with genetic structural variation

398 in SGT1, with two <100 bp regions often absent from parasites on sorghum but present for

399 parasites on maize and finger millet. We also identified a 59 bp contig with $96 \%$ similarity over

400 its full length to a transcript annotated as a pectin methylesterase; pectin methylesterases have

401 previously well-characterized functions in developing haustoria (Yang et al., 2014). The pectin

402 methylesterase transcript was not expressed in parasites grown on sorghum in most stages, but

403 exhibited low, non-zero expression in S. hermonthica seedlings after exposure to a haustorium

404 inducing factor (mean TPM = 0.03). We did not identify evidence for structural variation in this

405 gene in transcript-aligned reads, though strong differentiation between individuals from finger

406 millet $(\mathrm{AF}=0.65)$ and maize $(\mathrm{AF}=0.03)$ verified the signal observed from the $k$-mer association

407 analysis.

Two additional assembled host-differentiated contigs of 113 bp and 103 bp had $99 \%$ and

$40997 \%$ similarity, respectively, over their full length to separate regions of a single transcript

410 annotated as a precursor of chemocyanin (Table S2). Chemocyanins may be of particular

411 interest due to the evolutionary co-option of many pollen tube genes by parasitic plants for

412 haustorium development (Yang et al., 2014) and the key role of chemocyanin as an attractant

413 for directing pollen tube growth (Kim et al., 2003). The chemocyanin transcript was present in a

414 previously identified list of $S$. hermonthica "core parasitism" genes with highest expression in

415 stages 3 and/or 4 of haustorial development (Yang et al. 2014; Fig 3B). It was also highly

416 expressed in cells at the host-parasite interface in a study that used laser capture

417 microdissection to characterize gene expression in the S. hermonthica-sorghum interaction

418 (Honaas et al., 2013).

419 Alignment of our DNA sequences to the chemocyanin transcript reference revealed host420 associated structural variation (Fig. 3A). PCR-based confirmation indicated 500 bp or more of 
genome sequence directly upstream of the 5 ' end of the transcript was completely missing from

422 parasites on maize, suggesting potential impact on variation in gene expression levels. The

423 fragmented nature of the draft genome assembly precluded our ability to design PCR primers

424 completely spanning the deletion, but PCR banding patterns showed a characteristic absence of

425 the region in individuals having the allele more common on finger millet (Fig. S4), and a diversity

426 of deletion alleles at this site (Fig. S5). Using just sequences for individuals parasitizing finger

427 millet, we reconstructed a 934 bp contig, from reads that mapped to the transcript reference and

428 their unmapped pairs. Alignments to this 'finger millet' allele confirmed its presence in 9/12

429 individuals from Kisii parasitizing finger millet $(\mathrm{AF}=0.75)$ and $0 / 12$ parasites on maize (Table

430 S3). Among all individuals sequenced in our study, the allele is present at lowest frequency in

431 maize parasites $(\mathrm{AF}=0.14 ; n=35)$ and intermediate frequency for parasites on sorghum $(\mathrm{AF}=$

$432 \quad 0.22 ; n=18)$; and sugarcane $(\mathrm{AF}=0.33 ; n=3)$.

Validation of k-mer-based approaches-To further investigate signatures of selection

435 in the context of genome-wide patterns, we assembled a draft genome for $S$. hermonthica from

436 South Uganda. The total length of the assembled genome after filtering was 1,431 Mbp over

$43712,155,247$ contigs, with a maximum contig length of $37.5 \mathrm{~kb}$. The assembly was highly

438 fragmented with the largest 1.69 million contigs accounting for $50 \%$ of the assembly, and the 439 length of these contigs $\geq 110 \mathrm{bp}(\mathrm{N} / \mathrm{L} 50=1690935 / 110)$. Nevertheless, sequences of interest

440 were present on contigs long enough to allow for further interrogation. Specifically, the

441 assembled chemocyanin transcript for the finger millet allele had $97.8 \%$ identity over 918 bp to a

442 single contig of 1,055 bp (NODE_132909_length_1055_cov_32.632), and no other close hits.

443 After removing contigs shorter than $1 \mathrm{~kb}, 307026$ scaffolds with a total length of $479 \mathrm{Mbp}$

444 remained. A Tajima's D value of -1.7 in the Kisii population indicated a significant excess of low-

445 frequency polymorphism (empirical $P$-value $=0.009$ ) for the chemocyanin contig in the de novo 
assembly compared to the genome-wide value of $0.11 \pm 0.013$ (mean \pm std. error for nonoverlapping 1-kb windows; linear mixed effects model).

\section{DISCUSSION}

Agricultural weeds are increasingly recognized as important model systems for sequencing for parasitic plants, evolutionary analyses particularly for species with large, complex genomes (e.g. >1 Gb) remain a challenge (Lyko and Wicke, 2021). Consequently,

458 previous population-level diversity studies for $S$. hermonthica, one of the most damaging 459 parasitic plants in agriculture, have used reduced representation approaches (Unachukwu et al., 460 2017; Lopez et al., 2019). However, reduced representation approaches such as RAD-seq may 461 miss signatures of selection that are highly localized in the genome (Lowry et al., 2017; Lou et 462 al., 2021), and transcriptomes fail to provide information regarding non-coding regions of the 463 genome, which also generate phenotypic diversity. As the cost of sequencing continues to 464 decrease, whole genome resequencing coupled with alignment-free bioinformatic approaches 465 can provide a promising alternate approach for surveying population genomic diversity (Voichek 466 and Weigel, 2020). Here, we report some of the first publicly available WGS data for field467 sampled individuals of the parasitic weed Striga hermonthica. Our analyses underscore high 468 within-population genomic variation and implicate host-specific selection on genes involved 469 during parasite attachment and haustorial development.

470 Perhaps surprisingly, we find little genomic or phenotypic evidence for host-specific 471 selection on strigolactone perception variation in the studied populations. Specifically, one may 
472 expect selection on perception loci to be relatively strong, particularly since $S$. hermonthica is an

473 obligate parasite and so the costs of germination in the absence of a suitable host are high. The

474 genomes of Striga spp. include a diverse repertoire of strigolactone receptors, each with

475 variable affinity for different SLs (Tsuchiya et al., 2015; Yoshida et al., 2019), providing many

476 potential targets for selection on SL response variation. One possibility is that these receptors

477 are now subject to purifying selection in western Kenya, rather than diversifying selection

478 expected if SL perception variation is strongly linked to fitness variation across different hosts.

479 Environmental niche models from our previous study (Bellis et al., 2021) predict highest habitat

480 suitability for maize parasitism across sampling locations in our study (mean habitat suitability:

$4810.92)$ but lower suitability for sorghum and pearl millet ( 0.46 and 0.03 , respectively). Strong

482 selective pressure for maize parasitism may contribute to the generalist germination response

483 observed in western Kenya. Another possibility is that the particular host genotypes studied

484 here may overlap in SL exudation profile enough that selection on parasite germination rate

485 variation is not strong in these natural field populations. For example, while zealactones appear

486 to be uniquely produced by maize (Charnikhova et al., 2017), some varieties also naturally

487 produce sorgomol and 5-deoxystrigol in high quantities (Yoneyama et al., 2015), strigolactones

488 common in sorghum root exudate that promote strong germination response in S. hermonthica.

A third explanation is that SL perception is important for reciprocal selection and

490 coevolution only in some populations across the range of $S$. hermonthica. This explanation is

491 consistent with the idea of coevolutionary hotspots, where there is reciprocal selection among

492 coevolving species (Thompson, 2005), but with the genetic targets of selection involving

493 different stages of the infection process in different locations. In western Kenya, for example,

494 coevolutionary hotspots may be 'hotter' for genes involved in parasitic interactions post-

495 germination than for SL perception. Kenyan S. hermonthica populations exhibit a more

496 generalist germination response compared to populations from West Africa, which show

497 pronounced differences in response to orobanchol vs. 5-deoxystrigol from host root exudates or 
to strigolactone standards (Parker and Reid, 1979; Haussmann et al., 2004; Bellis et al., 2020).

499 This idea also corresponds with previous findings that East African S. hermonthica may have

500 greater average infestation success across diverse sorghum genotypes than West African

501 populations (Bozkurt et al., 2015). Functional $G_{H} \times G_{p}$ in strigolactone response variation may

502 segregate among populations in a different part of the parasite range than studied here, for

503 example among West African populations, or at a broader spatial scale, for example in West vs.

504 East African parasite populations (Haussmann et al., 2004; Bellis et al., 2020).

505 In contrast to general expectations (Nuismer and Dybdahl, 2016), our genomic analyses

506 revealed the strongest evidence for host-specific selection on genes involved in the later stages

507 of parasite development (Fig. 3, Fig. S3). The best evidence for host-specific selection on

508 haustorium loci came from our analyses of a transcript annotated as a chemocyanin precursor

509 (Fig. 3). In addition to strong differentiation between finger millet and maize hosts, the 1-kb

510 region including the chemocyanin exhibited an excess of rare polymorphism and multiple alleles

511 (Fig. S4-5), consistent with expectations for recurrent soft sweeps from standing genetic

512 variation (Pennings and Hermisson, 2006). Notably, even the strongest signals of host-specific

513 selection detected in our study did not reveal any loci exhibiting complete differentiation

514 between parasites on different hosts, indicating that there may be relatively few genetic barriers

515 to parasitism of different cereal host species in our region. The 'finger millet' chemocyanin allele,

516 for example, was also present at low frequency in the genomes of parasites on other host

517 species. This suggests a neutral impact of the allele on parasitism of other hosts, given that all

518 sequenced parasite individuals were already at an advanced life stage. The importance of

519 conditional neutrality for local adaptation in other systems (Lowry et al., 2019) further highlights

520 the complexity of selective pressures shaping local adaptation of parasitic plants to dynamic

521 host communities.

522

\section{CONCLUSIONS}


While our results emphasize the challenges of Striga management due to high genomic

525 diversity and adaptive potential, they also highlight the promise of low coverage WGS

526 approaches for functional genomics of non-model species. The outlier signal for two of the three

527 candidate loci we describe in detail here resulted from structural variation that would not have

528 been uncovered in an alignment-based analysis or using a RAD-Seq approach that may only

529 survey a small portion of the genome or be prone to allele drop-out. Reference-free approaches

530 continue to gain ground for studies of genomic and phenotypic variation in plants, with well-

531 documented advantages (VanWallendael and Alvarez, 2020; Voichek and Weigel, 2020). Our

532 study indicates that the utility of large WGS datasets may not be out of reach even for species

533 such as Striga hermonthica characterized by large, complex genomes. As the need to mitigate

534 biotic constraints on global food security becomes increasingly critical, reference-free analyses

535 coupled with WGS data may serve as a promising strategy for rapid characterization of alleles

536 involved in parasite adaptation across diverse environments.

\section{ACKNOWLEDGMENTS}

539 This study is based on work supported by a National Science Foundation Postdoctoral

540 Research Fellowship in Biology to E.S.B. under Grant 1711950, the Arkansas Biosciences

541 Institute (the major research component of the Arkansas Tobacco Settlement Proceeds Act of

542 2000), and the Emmy Noether-program of the German Science Foundation (WI4507/3-1 to

543 S.W.). A.K. and X.H. were supported by NSF EPSCoR Award 1946391. J.R.L. was supported

544 by NIH 1 R35 GM138300-01.

\section{AUTHOR CONTRIBUTIONS}

547 C.S.v.M., S.W., C.O.O., E.K., T.X., and E.S.B collected and processed samples and performed

548 laboratory experiments. C.S.v.M., S.W., A.K., and E.S.B. carried out analyses. X.H., S.W., 
549 S.M.R., C.W.D., J.R.L., and E.S.B. contributed to experimental design and interpretation. E.S.B.

550 and J.R.L. drafted the manuscript, with input and critical revision from all authors. All authors

551 approved the final version of the manuscript.

\section{DATA AND CODE AVAILABILITY}

554 Raw reads from whole genome sequencing of the 68 S. hermonthica individuals from the 2018

555 collection and the Ugandan reference have been deposited in the National Center for

556 Biotechnology Information (NCBI) Sequence Read Archive (SRA) database,

557 https://www.ncbi.nlm.nih.gov/sra (BioProject accession no. PRJNA801489). The reference

558 assembly of the Ugandan specimen is available for download and BLAST searches on WARPP

559 (https://warpp.app; (Kösters et al., 2021). Images associated with the different collection sites

560 are available from iNaturalist. Germination rate data and code to reproduce the analyses are

561 available at https://github.com/em-bellis/StrigaWGS.

\section{SUPPLEMENTARY INFORMATION}

563 Additional supporting information may be found online in the Supporting Information Section at

564 the end of the article.

565 Appendix S1: Supplementary Figures and Tables.

566

567 LITERATURE CITED

568 Akiyama, K., K. Matsuzaki, and H. Hayashi. 2005. Plant sesquiterpenes induce hyphal $569 \quad$ branching in arbuscular mycorrhizal fungi. Nature 435: 824-827.

570 Awad, A. A., D. Sato, D. Kusumoto, H. Kamioka, Y. Takeuchi, and K. Yoneyama. 2006.

571 Characterization of Strigolactones, Germination Stimulants for the Root Parasitic Plants 

Regulation 48: 221.

574 Bankevich, A., S. Nurk, D. Antipov, A. A. Gurevich, M. Dvorkin, A. S. Kulikov, V. M. Lesin, et al. 2012. SPAdes: a new genome assembly algorithm and its applications to single-cell sequencing. Journal of computational biology 19: 455-477.

Bates, D., M. Mächler, B. Bolker, and S. Walker. 2015. Fitting Linear Mixed-Effects Models Using Ime4. Journal of Statistical Software 67: 1-48.

Baucom, R. S. 2019. Evolutionary and ecological insights from herbicide-resistant weeds: what have we learned about plant adaptation, and what is left to uncover? New Phytologist

Bebawi, F. F., R. E. Eplee, C. E. Harris, and R. S. Norris. 1984. Longevity of witchweed (Striga 223: $68-82$.

584

Bellis, E., and E. Kelly. 2019. Striga hermonthica germination assay. protocols.io.

Bellis, E. S., E. A. Kelly, C. M. Lorts, H. Gao, V. L. DeLeo, G. Rouhan, A. Budden, et al. 2020. asiatica) seed. Weed Science 32: 494-497.

Bellis, E. S., C. M. McLaughlin, C. W. dePamphilis, and J. R. Lasky. 2021. The geography of parasite local adaptation to host communities. Ecography. sequence data. Bioinformatics 30: 2114-2120. 
Bolnick, D. I., L. H. Yang, J. A. Fordyce, J. M. Davis, and R. Svanbäck. 2002. Measuring Individual-Level Resource Specialization. Ecology 83: 2936-2941.

594 Bozkurt, M. L., P. Muth, H. K. Parzies, and B. I. G. Haussmann. 2015. Genetic diversity of East and West African Striga hermonthica populations and virulence effects on a contrasting set of sorghum cultivars. Weed Research 55: 71-81.

Bushnell, B. BBMap. Website sourceforge.net/projects/bbmap/.

Cardoso, C., T. Charnikhova, M. Jamil, P.-M. Delaux, F. Verstappen, M. Amini, D. Lauressergues, et al. 2014. Differential Activity of Striga hermonthica Seed Germination Stimulants and Gigaspora rosea Hyphal Branching Factors in Rice and Their Contribution to Underground Communication. PLOS ONE 9: 1-11.

Castellano, D., L. H. Uricchio, K. Munch, and D. Enard. 2019. Viruses rule over adaptation in conserved human proteins. bioRxiv: 555060.

Charnikhova, T. V., K. Gaus, A. Lumbroso, M. Sanders, J.-P. Vincken, A. D. Mesmaeker, C. P. Ruyter-Spira, et al. 2017. Zealactones. Novel natural strigolactones from maize.

Conn, C. E., R. Bythell-Douglas, D. Neumann, S. Yoshida, B. Whittington, J. H. Westwood, K. Shirasu, et al. 2015. Convergent evolution of strigolactone perception enabled host detection in parasitic plants. Science 349: 540-543. Host lignin composition affects haustorium induction in the parasitic plants 
613

614

615

616

617

618

619

620

621

622

623

624

625

626

627

628

629

630

631

632

633

634

Dayou, O., W. Kibet, P. Ojola, P. I. Gangashetty, S. Wicke, and S. Runo. 2021. Two-tier witchweed (Striga hermonthica) resistance in wild pearl millet (Pennisetum glaucum) 29Aw. Weed Science 69: 300-306.

Ebert, D., and P. D. Fields. 2020. Host-parasite co-evolution and its genomic signature. Nature Reviews Genetics 21: 754-768.

Ejeta, G. 2007. The Striga scourge in Africa: a growing pandemic. Integrating new technologies for Striga control: towards ending the witch-hunt, 3-16. World Scientific.

Estep, M. C., B. S. Gowda, K. Huang, M. P. Timko, and J. L. Bennetzen. 2012. Genomic characterization for parasitic weeds of the genus Striga by sample sequence analysis. The Plant Genome 5.

Fumagalli, M., M. Sironi, U. Pozzoli, A. Ferrer-Admettla, L. Pattini, and R. Nielsen. 2011. Signatures of environmental genetic adaptation pinpoint pathogens as the main selective pressure through human evolution. PLoS genetics 7: e1002355.

Gbèhounou, G., A. H. Pieterse, and J. A. Verkleij. 2003. Longevity of Striga seeds reconsidered: results of a field study on purple witchweed (Striga hermonthica) in Benin. Weed Science 51: 940-946.

Gethi, J. G., M. E. Smith, S. E. Mitchell, and S. Kresovich. 2005. Genetic diversity of Striga hermonthica and Striga asiatica populations in Kenya. Weed Research 45: 64-73.

Gobena, D., M. Shimels, P. J. Rich, C. Ruyter-Spira, H. Bouwmeester, S. Kanuganti, T. Mengiste, and G. Ejeta. 2017. Mutation in sorghum LOW GERMINATION STIMULANT 1 alters strigolactones and causes Striga resistance. Proceedings of the National Academy of Sciences 114: 4471-4476. 
Gurevich, A., V. Saveliev, N. Vyahhi, and G. Tesler. 2013. QUAST: quality assessment tool for genome assemblies. Bioinformatics 29: 1072-1075.

Hall, M. D., G. Bento, and D. Ebert. 2017. The evolutionary consequences of stepwise infection processes. Trends in Ecology \& Evolution 32: 612-623.

Hall, M. D., J. Routtu, and D. Ebert. 2019. Dissecting the genetic architecture of a stepwise infection process. Molecular ecology 28: 3942-3957.

641 Haussmann, B., D. Hess, G. Omanya, R. Folkertsma, B. Reddy, M. Kayentao, H. Welz, and H. Geiger. 2004. Genomic regions influencing resistance to the parasitic weed Striga hermonthica in two recombinant inbred populations of sorghum. Theoretical and Applied

Honaas, L. A., E. K. Wafula, Z. Yang, J. P. Der, N. J. Wickett, N. S. Altman, C. G. Taylor, et al. Genetics 109: 1005-1016.

649 Ichihashi, Y., M. Kusano, M. Kobayashi, K. Suetsugu, S. Yoshida, T. Wakatake, K. Kumaishi, et al. 2017. Transcriptomic and Metabolomic Reprogramming from Roots to Haustoria in

652 Jackman, S. D., B. P. Vandervalk, H. Mohamadi, J. Chu, S. Yeo, S. A. Hammond, G. Jahesh, et 653 al. 2017. ABySS 2.0: resource-efficient assembly of large genomes using a Bloom filter. Genome research 27: 768-777. 
Kavuluko, J., M. Kibe, I. Sugut, W. Kibet, J. Masanga, S. Mutinda, M. Wamalwa, et al. 2020. GWAS provides biological insights into mechanisms of the parasitic plant (Striga) resistance in Sorghum.

Kim, G., M. L. LeBlanc, E. K. Wafula, C. W. DePamphilis, and J. H. Westwood. 2014. Genomicscale exchange of mRNA between a parasitic plant and its hosts. Science 345: 808-

Kim, S., J.-C. Mollet, J. Dong, K. Zhang, S.-Y. Park, and E. M. Lord. 2003. Chemocyanin, a small basic protein from the lily stigma, induces pollen tube chemotropism. Proceedings of the National Academy of Sciences 100: 16125-16130.

Kim, S. Y., K. E. Lohmueller, A. Albrechtsen, Y. Li, T. Korneliussen, G. Tian, N. Grarup, et al. 2011. Estimation of allele frequency and association mapping using next-generation sequencing data. BMC Bioinformatics 12: 231.

Korneliussen, T. S., A. Albrechtsen, and R. Nielsen. 2014. ANGSD: Analysis of Next Generation Sequencing Data. BMC Bioinformatics 15: 356.

669 Kösters, L. M., S. Wiechers, P. Lyko, K. F. Müller, and S. Wicke. 2021. WARPP—web application for the research of parasitic plants. Plant Physiology 185: 1374-1380.

671 Li, H. 2013. Aligning sequence reads, clone sequences and assembly contigs with BWA-MEM.

672 Li, H., B. Handsaker, A. Wysoker, T. Fennell, J. Ruan, N. Homer, G. Marth, et al. 2009. The

673 Sequence Alignment/Map format and SAMtools. Bioinformatics 25: 2078-2079.

674 Li, J., and M. P. Timko. 2009. Gene-for-gene resistance in Striga-cowpea associations. Science 325: 1094-1094. 
676 Liu, N., G. Shen, Y. Xu, H. Liu, J. Zhang, S. Li, J. Li, et al. 2020. Extensive inter-plant protein transfer between Cuscuta parasites and their host plants. Molecular plant 13: 573-585.

678 Lopez, L., E. S. Bellis, E. Wafula, S. J. Hearne, L. Honaas, P. E. Ralph, M. P. Timko, et al. 2019. Transcriptomics of host-specific interactions in natural populations of the parasitic plant purple witchweed (Striga hermonthica). Weed Science 67: 397-411.

Lou, R., A. Jacobs, A. Wilder, and N. O. Therkildsen. 2021. A beginner's guide to low-coverage whole genome sequencing for population genomics. Authorea.

Louthan, A. M., and K. M. Kay. 2011. Comparing the adaptive landscape across trait types: larger QTL effect size in traits under biotic selection. BMC evolutionary biology 11: 1-12.

Lowry, D. B., S. Hoban, J. L. Kelley, K. E. Lotterhos, L. K. Reed, M. F. Antolin, and A. Storfer. 2017. Breaking RAD: an evaluation of the utility of restriction site-associated DNA

Lowry, D. B., J. T. Lovell, L. Zhang, J. Bonnette, P. A. Fay, R. B. Mitchell, J. Lloyd-Reilley, et al. 2019. QTL × environment interactions underlie adaptive divergence in switchgrass across a large latitudinal gradient. Proceedings of the National Academy of Sciences gene losses alongside global genome size inflation and gene births. Plant Physiology 186: 1412-1423. 
Mallu, T. S., S. Mutinda, S. M. Githiri, D. Achieng Odeny, and S. Runo. 2021. New preattachment Striga resistant sorghum adapted to African agro-ecologies. Pest Management Science 77: 2894-2902.

Masumoto, N., Y. Suzuki, S. Cui, M. Wakazaki, M. Sato, K. Kumaishi, A. Shibata, et al. 2021. Three-dimensional reconstructions of haustoria in two parasitic plant species in the Orobanchaceae. Plant Physiology 185: 1429-1442.

Mbuvi, D. A., C. W. Masiga, E. K. Kuria, J. Masanga, M. Wamalwa, A. Mohamed, D. Odeny, et al. 2017. Novel sources of witchweed (Striga) resistance from wild sorghum accessions. Frontiers in plant science 8: 116.

Mutinda, S. M., J. Masanga, J. M. Mutuku, S. Runo, and A. Alakonya. 2018. KSTP 94, an openpollinated maize variety has postattachment resistance to purple witchweed (Striga hermonthica). Weed science 66: 525-529.

Nei, M. 1973. Analysis of Gene Diversity in Subdivided Populations. Proceedings of the National Academy of Sciences 70: 3321-3323.

Nei, M., and R. K. Chesser. 1983. Estimation of fixation indices and gene diversities. Annals of Human Genetics 47: 253-259.

712 Nelson, D. C. 2021. The mechanism of host-induced germination in root parasitic plants. Plant $713 \quad$ Physiology 185: 1353-1373.

714 Nuismer, S. L., and M. F. Dybdahl. 2016. Quantifying the coevolutionary potential of multistep 715 immune defenses. Evolution 70: 282-295. 
716 Ondov, B. D., T. J. Treangen, P. Melsted, A. B. Mallonee, N. H. Bergman, S. Koren, and A. M. Phillippy. 2016. Mash: fast genome and metagenome distance estimation using MinHash. Genome Biology 17: 132.

Padgham, M., and M. D. Sumner. 2021. geodist: Fast, Dependency-Free Geodesic Distance Calculations.

721 Paradis, E., and K. Schliep. 2019. ape 5.0: an environment for modern phylogenetics and evolutionary analyses in R. Bioinformatics 35: 526-528.

Parker, C., and D. C. Reid. 1979. Host specificity in Striga species: Some preliminary observations. Proceeding of the Second International Symposium on Parasitic Weeds,

Pennings, P. S., and J. Hermisson. 2006. Soft sweeps III: the signature of positive selection Raleigh, NC, 1979,.

Poland, J. A., P. J. Balint-Kurti, R. J. Wisser, R. C. Pratt, and R. J. Nelson. 2009. Shades of gray: the world of quantitative disease resistance. Trends in plant science 14: 21-29.

R Core Team. 2020. R: A language and environment for statistical computing. R Foundation for Statistical Computing, Vienna, Austria.

Rahman, A., I. Hallgrímsdóttir, M. Eisen, and L. Pachter. 2018. Association mapping from sequencing reads using k-mers J. Flint [ed.],. eLife 7: e32920.

734 Ravensdale, M., A. Nemri, P. H. Thrall, J. G. Ellis, and P. N. Dodds. 2011. Co-evolutionary interactions between host resistance and pathogen effector genes in flax rust disease.

$736 \quad$ Molecular plant pathology 12: 93-102. 
737 Savary, S., L. Willocquet, S. J. Pethybridge, P. Esker, N. McRoberts, and A. Nelson. 2019. The global burden of pathogens and pests on major food crops. Nature ecology \& evolution 3: $430-439$.

Shahid, S., G. Kim, N. R. Johnson, E. Wafula, F. Wang, C. Coruh, V. Bernal-Galeano, et al. 2018. MicroRNAs from the parasitic plant Cuscuta campestris target host messenger RNAs. Nature 553: 82-85.

Shen, G., N. Liu, J. Zhang, Y. Xu, I. T. Baldwin, and J. Wu. 2020. Cuscuta australis (dodder) parasite eavesdrops on the host plants' FT signals to flower. Proceedings of the National Academy of Sciences 117: 23125-23130.

Spallek, T., M. Mutuku, and K. Shirasu. 2013. The genus Striga: a witch profile. Molecular plant pathology 14: 861-869.

748 Therkildsen, N. O., and S. R. Palumbi. 2017. Practical low-coverage genome-wide sequencing 749 of hundreds of individually barcoded samples for population and evolutionary genomics in nonmodel species. Molecular Ecology Resources 17: 194-208.

751 Thompson, J. N. 2005. The geographic mosaic of coevolution. University of Chicago Press.

752 Thrall, P. H., A.-L. Laine, M. Ravensdale, A. Nemri, P. N. Dodds, L. G. Barrett, and J. J. Burdon.

753 2012. Rapid genetic change underpins antagonistic coevolution in a natural host-

754 pathogen metapopulation. Ecology letters 15: 425-435.

755 Timko, M. P., K. Huang, and K. E. Lis. 2012. Host resistance and parasite virulence in Striga756 host plant interactions: A shifting balance of power. Weed Science 60: 307-315. 
Toh, S., D. Holbrook-Smith, P. J. Stogios, O. Onopriyenko, S. Lumba, Y. Tsuchiya, A. Savchenko, and P. McCourt. 2015. Structure-function analysis identifies highly sensitive strigolactone receptors in Striga. Science 350: 203-207.

Tsuchiya, Y., M. Yoshimura, Y. Sato, K. Kuwata, S. Toh, D. Holbrook-Smith, H. Zhang, et al. 2015. Probing strigolactone receptors in Striga hermonthica with fluorescence. Science

Unachukwu, N. N., A. Menkir, I. Y. Rabbi, M. Oluoch, S. Muranaka, A. Elzein, G. Odhiambo, et al. 2017. Genetic diversity and population structure of Striga hermonthica populations from Kenya and Nigeria. Weed Research 57: 293-302.

VanWallendael, A., and M. Alvarez. 2020. Alignment-free methods for polyploid genomes: quick and reliable genetic distance estimation. bioRxiv.

Vigueira, C. C., K. M. Olsen, and A. L. Caicedo. 2013. The red queen in the corn: agricultural weeds as models of rapid adaptive evolution. Heredity 110: 303-311.

Voichek, Y., and D. Weigel. 2020. Identifying genetic variants underlying phenotypic variation in plants without complete genomes. Nature Genetics 52: 534-540.

Westwood, J. H., C. W. dePamphilis, M. Das, M. Fernández-Aparicio, L. A. Honaas, M. P. Timko, E. K. Wafula, et al. 2012. The Parasitic Plant Genome Project: New Tools for

775 Wicke, S., K. F. Müller, C. W. dePamphilis, D. Quandt, S. Bellot, and G. M. Schneeweiss. 2016. 776 Mechanistic model of evolutionary rate variation en route to a nonphotosynthetic lifestyle 777 in plants. Proceedings of the National Academy of Sciences 113: 9045-9050. 
778

779

780

781

782

783

784

785

786

787

788

789

790

791

792

793

794

795

796

797

798

799

Wicke, S., K. F. Müller, C. W. de Pamphilis, D. Quandt, N. J. Wickett, Y. Zhang, S. S. Renner, and G. M. Schneeweiss. 2013. Mechanisms of functional and physical genome reduction in photosynthetic and nonphotosynthetic parasitic plants of the broomrape family. The Plant Cell 25: 3711-3725.

Wilfert, L., and P. Schmid-Hempel. 2008. The genetic architecture of susceptibility to parasites. BMC Evolutionary Biology 8: 1-8.

Wood, D. E., J. Lu, and B. Langmead. 2019. Improved metagenomic analysis with Kraken 2. Genome biology 20: 1-13.

Yang, Z., E. K. Wafula, L. A. Honaas, H. Zhang, M. Das, M. Fernandez-Aparicio, K. Huang, et al. 2014. Comparative Transcriptome Analyses Reveal Core Parasitism Genes and Suggest Gene Duplication and Repurposing as Sources of Structural Novelty. Molecular Biology and Evolution 32: 767-790.

Yang, Z., E. K. Wafula, G. Kim, S. Shahid, J. R. McNeal, P. E. Ralph, P. R. Timilsena, et al. 2019. Convergent horizontal gene transfer and cross-talk of mobile nucleic acids in parasitic plants. Nature Plants 5: 991-1001.

Yoneyama, K., R. Arakawa, K. Ishimoto, H. I. Kim, T. Kisugi, X. Xie, T. Nomura, et al. 2015. Difference in Striga-susceptibility is reflected in strigolactone secretion profile, but not in compatibility and host preference in arbuscular mycorrhizal symbiosis in two maize cultivars. New Phytologist 206: 983-989.

Yoneyama, K., X. Xie, H. Sekimoto, Y. Takeuchi, S. Ogasawara, K. Akiyama, H. Hayashi, and K. Yoneyama. 2008. Strigolactones, host recognition signals for root parasitic plants and arbuscular mycorrhizal fungi, from Fabaceae plants. New Phytologist 179: 484-494. 
800 Yoshida, S., J. K. Ishida, N. M. Kamal, A. M. Ali, S. Namba, and K. Shirasu. 2010. A full-length enriched cDNA library and expressed sequence tag analysis of the parasitic weed, Striga hermonthica. BMC Plant Biology 10: 1-10.

803 Yoshida, S., S. Kim, E. K. Wafula, J. Tanskanen, Y.-M. Kim, L. Honaas, Z. Yang, et al. 2019.

804 Genome sequence of Striga asiatica provides insight into the evolution of plant 805 parasitism. Current Biology 29: 3041-3052.

806

807 

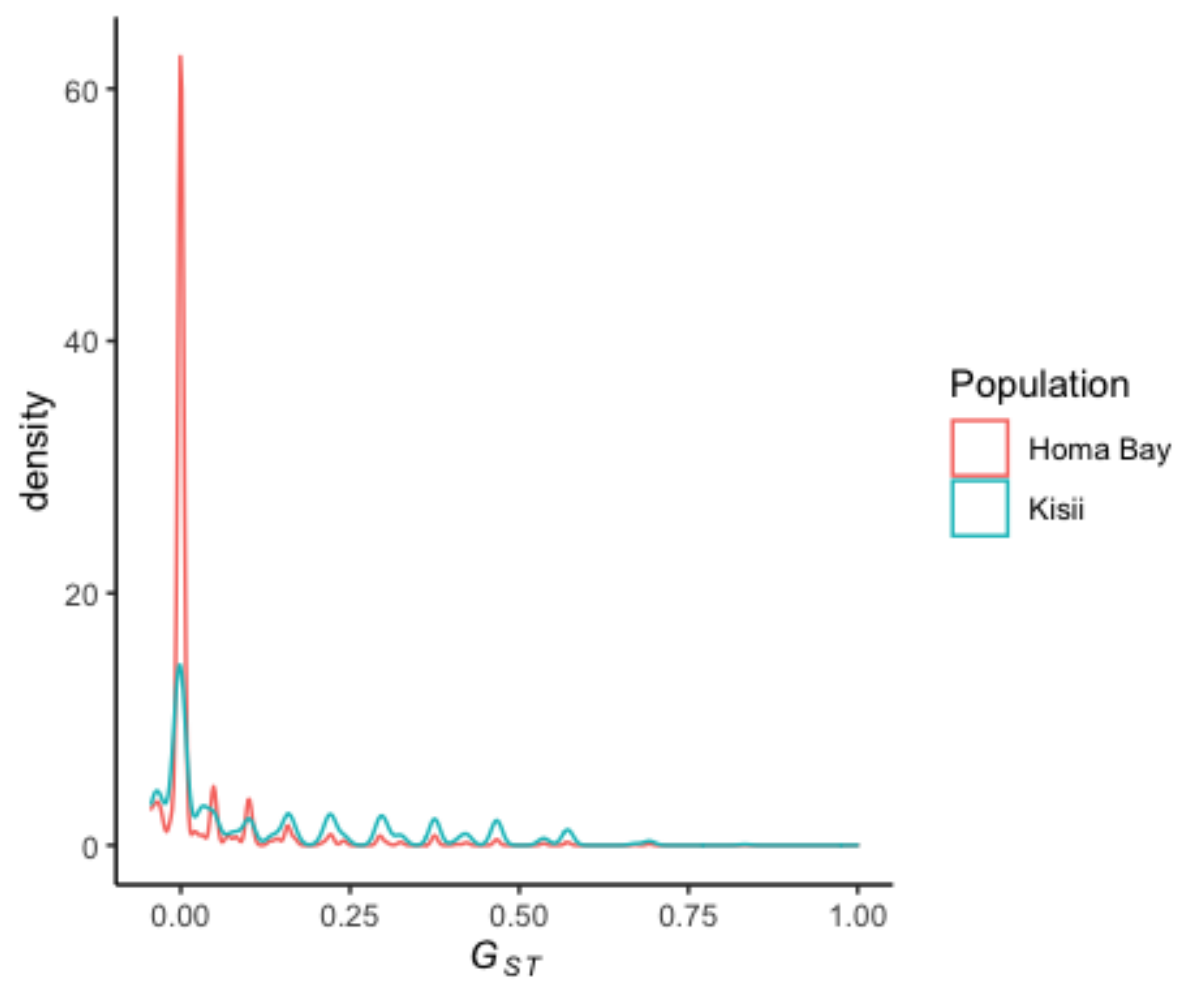

811 Figure S1. Distribution of $G_{S T}$ values. For Homa Bay, $G_{S T}$ is based on comparisons for

812 parasites on adjacent plots of maize vs. sorghum at 2,765,562 31-mers. For Kisii, $G_{S T}$ is based

813 on comparisons for parasites on adjacent plots of maize vs. sorghum at 2,123,902 31-mers. 


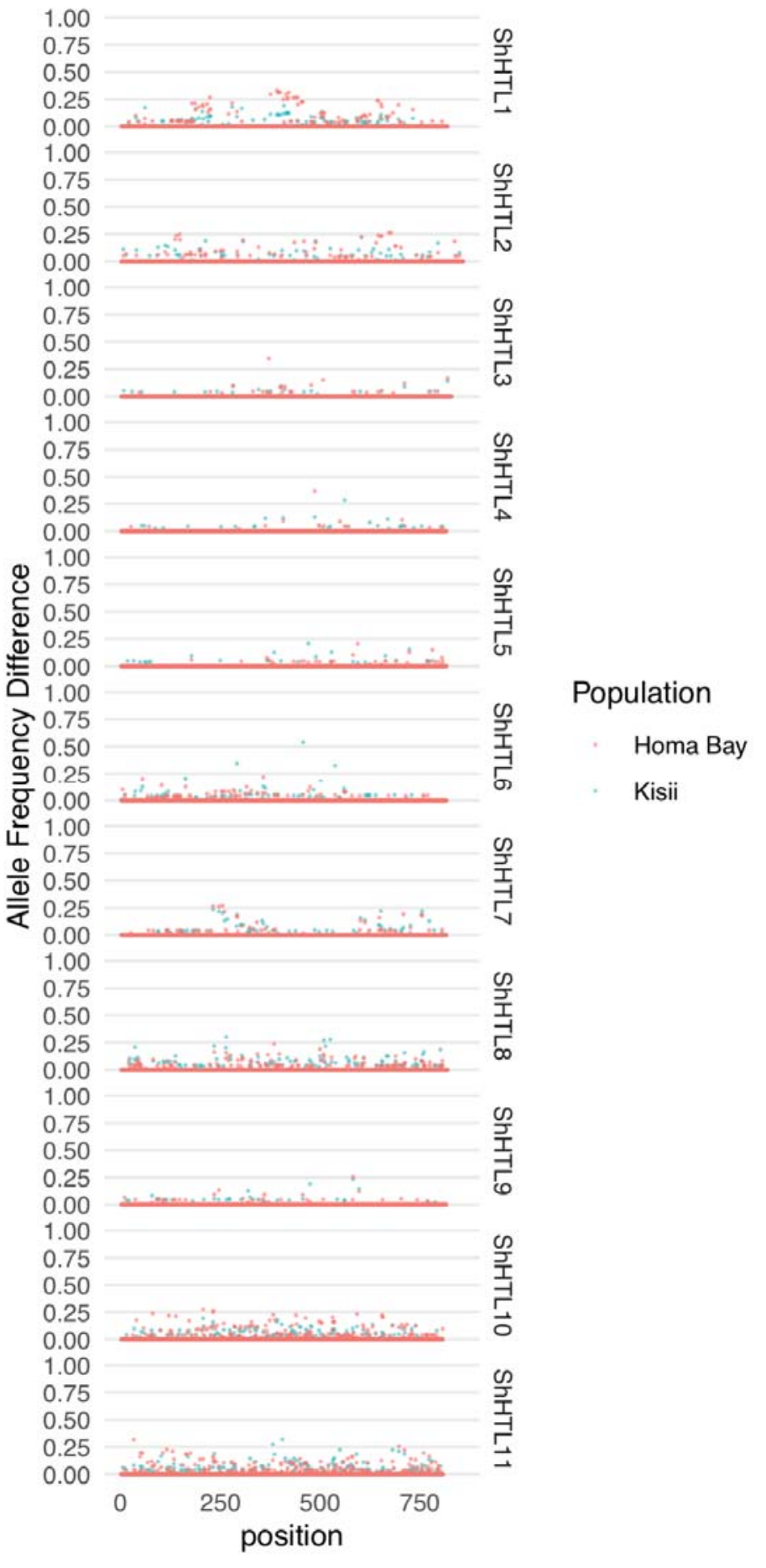

818 Figure S2. Allele frequency differences for 11 ShHTL receptors from Tsuchiya et al. (2015).

819 Allele frequencies were estimated from genotype likelihoods based on reads mapped to each

820 reference transcript. Allele frequency was estimated for parasites on each host species 
821 separately ( $n=12$ per unique host and population). The difference in allele frequency estimates 822 between two hosts within a single population is shown.

823

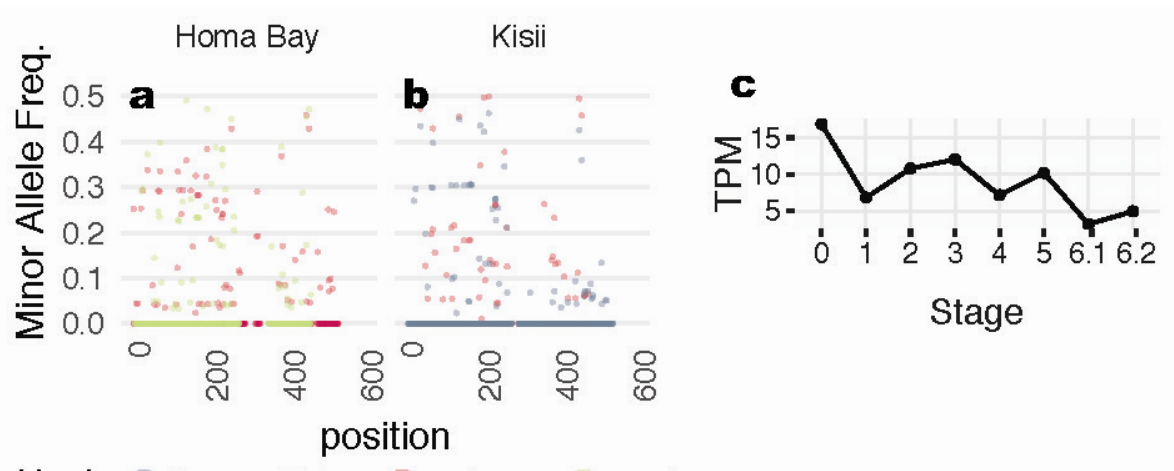

Host finger millet maize sorghum

825 Figure S3. $(a, b)$ Minor allele frequency differences based on alignments to the SGT1 transcript

826 sequence (StHeBC4_p_c12587_g2_i1), split by population. If the locus is not present in any of

827 the sequenced individuals for that population due to genetic structural variation, no data point is

828 shown. Frequencies were estimated based on genotype likelihoods from $n=12$ individuals per

829 unique host and population. (c) Gene expression data in transcripts per million (TPM) from the

830 PPGPII data across the 6 stages of haustorial development from data published. 


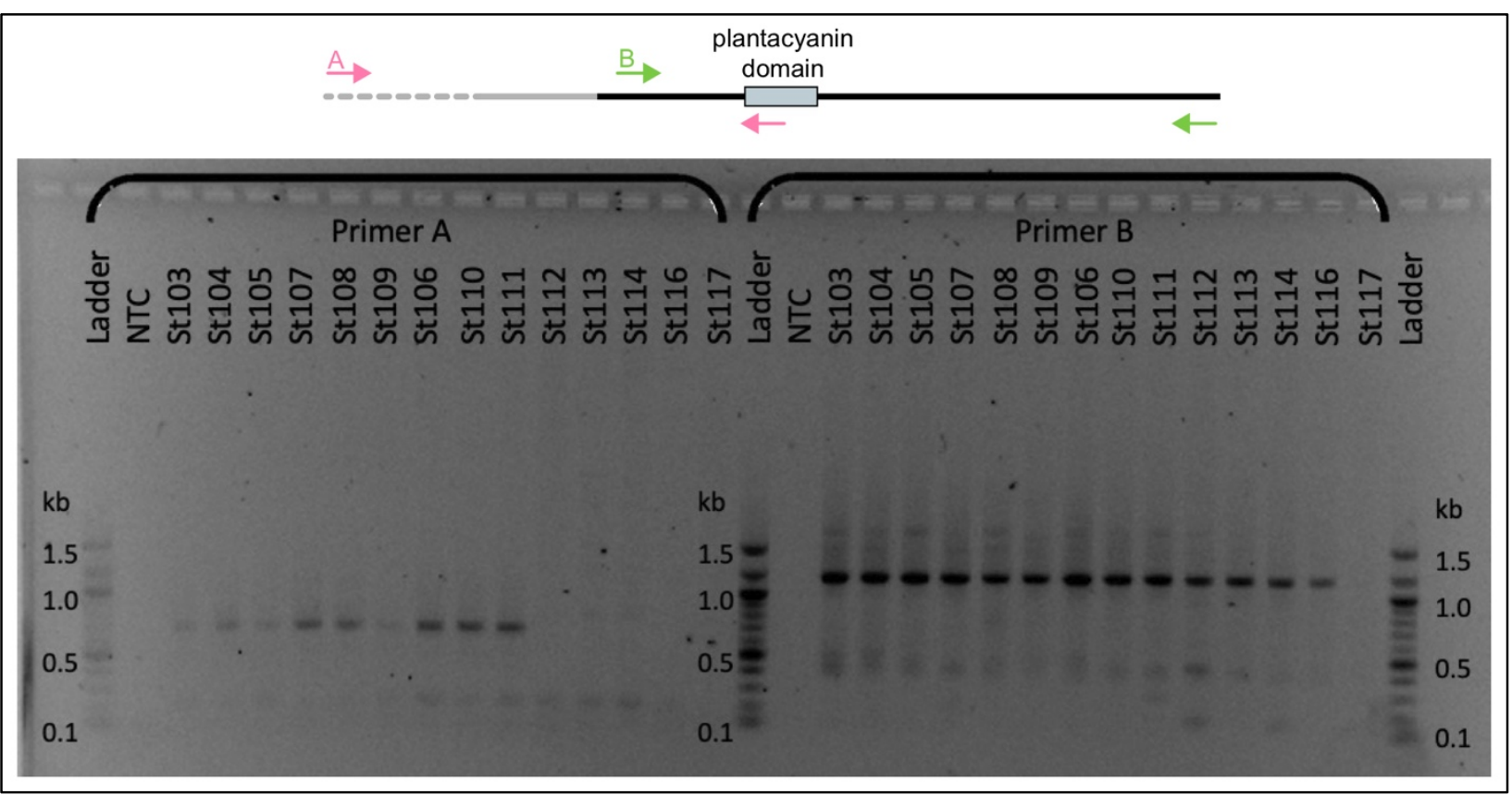

Figure S4. PCR banding results to confirm chemocyanin deletion calls. In the top panel,

834 binding sites for primer sets $A$ (pink) and B (green) are shown relative to location of the

835 transcript sequence (solid black line), location of the deletion (grey dashed line), and conserved

836 plantacyanin domain (light blue rectangle). Primer set A does not yield a 676-bp band for

837 individuals with the deletion (lanes 11-15) whereas primer set B amplifies a region downstream

838 of the deletion. NTC: no template control. 


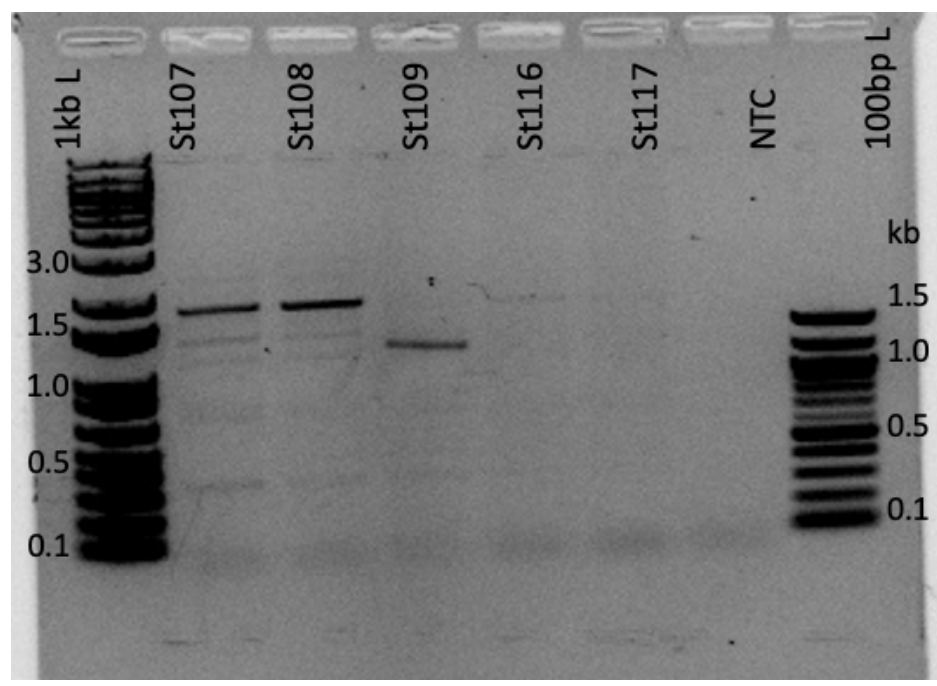

841 Figure S5. PCR banding results suggest multiple deletion variants. PCR amplification

842 using the outer primers from set A (5'-TCTCGATCCTTTTGGAATGG-3') and B (5'-

843 TGGGGAAAGAGGTAGTGCAA-3'); see Fig. S4). NTC: no template control. Lane 1: Sh107;

844 Lane 2: Sh108, Lane 3: Sh109; Lane 4: Sh116; Lane 5: Sh117.

845

846 
847 Table S1. GLMM for analysis of germination rate variation

\begin{tabular}{lcccc}
\hline & Estimate & Std. Error & z-value & $\boldsymbol{P}$ \\
\hline Intercept & 2.29 & 0.97 & 2.37 & 0.017 \\
Site $==$ Kisii & -2.16 & 0.79 & -2.72 & 0.006 \\
Host == Maize & -1.13 & 0.79 & -1.43 & 0.153 \\
Host == Sorghum & -1.90 & 1.12 & -1.70 & 0.089 \\
Treatment == ORO & -0.16 & 0.06 & -2.85 & 0.004
\end{tabular}

848

849

850 
851 Table S2. Hits to transcripts from the Parasitic Plant Genome Project (PPGPII) or Sh14v2 (from

852 Yoshida 2019) for contigs assembled from host-associated $k$-mers for Striga hermontica Homa

853 Bay population (maize vs. sorghum hosts). Transcripts with greater than $85 \%$ identity over at

854 least 45 bp are shown.

\begin{tabular}{lll}
\hline PGPPII & Sh14v2 & Description \\
\hline StHeBC4_p_c26811_g0_i3 & Sh14Contig_5850 & lipid-a-disaccharide synthase-like \\
None & Sh14Contig_37747 & None \\
None & Sh14Contig_44696 & None \\
StHeBC4_p_c12903_g35328_i2 & Sh14Contig_229 & retrotransposon gag protein \\
StHeBC4_p_c12587_g2_i1 & Sh14Contig_28742 & disease-resistance protein SGT1 \\
None & Sh14Contig_5301 & None \\
None & Sh14Contig_37747 & None \\
None & Sh14Contig_60980 & hypothetical protein ASPNIDRAFT_144168 \\
StHeBC4_u_c25350_g11_i1 & Sh14Contig_78939 & None \\
None & Sh14Contig_41198 & None \\
None & Sh14Contig_32559 & None \\
StHeBC4_u_c12903_g30818_i7 & Sh14Contig_35967 & None \\
None & Sh14Contig_47805 & None \\
StHeBC4_p_c9824_g0_i1 & None & None \\
StHeBC4_p_c12903_g42998_i1 & None & None \\
\hline
\end{tabular}


Table S3. Hits to transcripts from the Parasitic Plant Genome Project (PPGPII) or Sh14v2 (from Yoshida 2019) for contigs assembled from host-associated $k$-mers for Striga hermontica Kisii

858 population (maize vs. finger millet hosts). Transcripts with greater than $85 \%$ identity over at least

$85945 \mathrm{bp}$ are shown.

\begin{tabular}{|c|c|c|}
\hline PGPPII & Sh14v2 & Description \\
\hline None & Sh14Contig_45649 & None \\
\hline StHeBC4_u_c12903_g32907_i4 & Sh14Contig_20178 & $\begin{array}{l}\text { probable nicotinate-nucleotide } \\
\text { pyrophosphorylase }\end{array}$ \\
\hline StHeBC4_u_c12903_g22013_i6 & Sh14Contig_24947 & None \\
\hline StHeBC4_u_c3941_g0_i1 & Sh14Contig_42609 & hypothetical protein VITISV_012016 \\
\hline None & Sh14Contig_70490 & None \\
\hline StHeBC4_u_c12903_g34431_i1 & Sh14Contig_17925 & upf0326 protein at4g17486-like \\
\hline StHeBC4_p_c12903_g35474_i1 & Sh14Contig_4481 & None \\
\hline StHeBC4_u_c24490_g2_i1 & Sh14Contig_81114 & None \\
\hline StHeBC4_p_c12903_g14638_i24 & 4Sh14Contig_23122 & far-red impaired response protein \\
\hline StHeBC4_u_c12903_g34431_i2 & Sh14Contig_17925 & upf0326 protein at4g17486-like \\
\hline StHeBC4_u_c12903_g19156_i3 & Sh14Contig_3012 & alcohol dehydrogenase homolog \\
\hline StHeBC4_p_c26043_g0_i2 & Sh14Contig_13886 & omega-amidase nit2 \\
\hline StHeBC4_p_c12903_g10585_i1 & Sh14Contig_34611 & None \\
\hline None & Sh14Contig_20326 & None \\
\hline StHeBC4_p_c12903_g6756_i1 & Sh14Contig_12790 & None \\
\hline StHeBC4_u_c12903_g15860_i5 & Sh14Contig_29096 & None \\
\hline StHeBC4_u_c12903_g7220_i1 & Sh14Contig_60050 & None \\
\hline None & Sh14Contig_3989 & None \\
\hline StHeBC4_p_c12903_g2276_i2 & Sh14Contig_4881 & $\begin{array}{l}\text { rna polymerase ii c-terminal domain } \\
\text { phosphatase-like } 1 \text {-like }\end{array}$ \\
\hline StHeBC4_u_c12903_g1840_i3 & Sh14Contig_19406 & e3 ubiquitin-protein ligase ring1-like \\
\hline None & Sh14Contig_71030 & None \\
\hline StHeBC4_p_c12903_g39698_i9 & Sh14Contig_45458 & gag-protease polyprotein \\
\hline StHeBC4_u_c12903_g32907_i12 & 2Sh14Contig_81511 & None \\
\hline StHeBC4_u_c12903_g34427_i1 & Sh14Contig_46360 & None \\
\hline StHeBC4_p_c12903_g35474_i1 & Sh14Contig_4481 & None \\
\hline StHeBC4_p_c12903_g11444_i1 & Sh14Contig_40193 & polyprotein \\
\hline StHeBC4_u_c19051_g0_i1 & Sh14Contig_66323 & None \\
\hline StHeBC4_p_c12903_g10585_i1 & Sh14Contig_12790 & None \\
\hline StHeBC4_u_c12903_g1736_i4 & Sh14Contig_28260 & None \\
\hline None & Sh14Contig_31117 & $\begin{array}{l}\text { PREDICTED: uncharacterized protein } \\
\text { LOC100256114 }\end{array}$ \\
\hline StHeBC4_u_c12903_g33489_i1 & Sh14Contig_51019 & None \\
\hline StHeBC4_p_c16693_g0_i2 & Sh14Contig_33691 & hypothetical protein VITISV_018984 \\
\hline StHeBC4_p_c23323_g2_i3 & Sh14Contig_3290 & diphthamide biosynthesis protein 2-like \\
\hline StHeBC4_p_c18975_g7_i10 & Sh14Contig_7369 & None \\
\hline StHeBC4_p_c12903_g4626_i6 & Sh14Contig_4698 & retrotransposon protein \\
\hline None & Sh14Contig_80867 & None \\
\hline StHeBC4_h_c11261_g0_i2 & Sh14Contig_13520 & chemocyanin precursor \\
\hline StHeBC4_p_c23585_g1_i4 & Sh14Contig_1911 & None \\
\hline StHeBC4_p_c24979_g0_i1 & Sh14Contig_4792 & $97 \mathrm{kda}$ heat shock protein \\
\hline StHeBC4_h_c12903_g23955_i1 & Sh14Contig_45458 & gag-protease polyprotein \\
\hline
\end{tabular}




\begin{tabular}{|c|c|c|}
\hline StHeBC4_u_c12903_g1547_i1 & Sh14Contig_68469 & None \\
\hline None & Sh14Contig_48379 & None \\
\hline StHeBC4_p_c12903_g30160_i1 & Sh14Contig_57886 & None \\
\hline None & Sh14Contig_64011 & None \\
\hline None & Sh14Contig_1642 & retrotransposon ty1-copia subclass \\
\hline StHeBC4_p_c22011_g3_i2 & Sh14Contig_15531 & wd repeat-containing protein 26 -like \\
\hline StHeBC4_p_c12903_g7195_i9 & Sh14Contig_60490 & unnamed protein product \\
\hline StHeBC4_h_c24484_g1_i2 & Sh14Contig_42033 & hypothetical protein VITISV_042364 \\
\hline StHeBC4_p_c20621_g7_i3 & Sh14Contig_4748 & gag-pol precursor \\
\hline StHeBC4_u_c12903_g27039_i4 & Sh14Contig_4713 & pectin methylesterase \\
\hline StHeBC4_p_c18975_g7_i5 & Sh14Contig_7369 & None \\
\hline None & Sh14Contig_65419 & None \\
\hline StHeBC4_u_c12903_g32907_i15 & Sh14Contig_29096 & None \\
\hline None & Sh14Contig_52781 & None \\
\hline StHeBC4_p_c12903_g208_i2 & Sh14Contig_7920 & None \\
\hline StHeBC4_p_c12903_g22741_i4 & Sh14Contig_9532 & pattern formation \\
\hline StHeBC4_p_c12903_g38175_i2 & Sh14Contig_17588 & ring zinc finger ankyrin protein \\
\hline None & Sh14Contig_4395 & unnamed protein product [Vitis vinifera] \\
\hline StHeBC4_u_c12903_g34427_i3 & Sh14Contig_46360 & None \\
\hline StHeBC4_h_c11261_g0_i1 & Sh14Contig_13520 & chemocyanin precursor \\
\hline StHeBC4_h_c12903_g40212_i2 & Sh14Contig_24771 & copia Itr rider \\
\hline StHeBC4_p_c13584_g0_i1 & Sh14Contig_33358 & None \\
\hline StHeBC4_u_c12903_g16409_i3 & Sh14Contig_576 & atp-dependent helicase brm-like \\
\hline StHeBC4_h_c11483_g0_i1 & Sh14Contig_55439 & FAR1; Zinc finger, SWIM-type \\
\hline StHeBC4_p_c12903_g20722_i11 & Sh14Contig_28640 & $\begin{array}{l}\text { PREDICTED: uncharacterized protein } \\
\text { LOC100854178, partial }\end{array}$ \\
\hline StHeBC4_h_c12903_g12703_i2 & Sh14Contig_9184 & photosystem ii cp43 chlorophyll apoprotein \\
\hline StHeBC4_p_c12903_g2185_i1 & Sh14Contig_12790 & None \\
\hline StHeBC4_p_c12903_g34644_i1 & Sh14Contig_4585 & trehalose-phosphatase synthase 2 \\
\hline StHeBC4_p_c24106_g0_i1 & Sh14Contig_40210 & None \\
\hline StHeBC4_h_c18968_g1_i5 & Sh14Contig_1642 & retrotransposon ty1-copia subclass \\
\hline StHeBC4_u_c3088_g0_i1 & Sh14Contig_31622 & None \\
\hline StHeBC4_u_c12903_g35782_i1 & Sh14Contig_27773 & None \\
\hline None & Sh14Contig_23637 & None \\
\hline StHeBC4_u_c19051_g0_i1 & Sh14Contig_63267 & None \\
\hline None & Sh14Contig_11046 & uncharacterized protein loc100253271 \\
\hline StHeBC4_u_c12903_g27691_i1 & Sh14Contig_35466 & None \\
\hline StHeBC4_u_c22214_g0_i6 & Sh14Contig_43769 & None \\
\hline StHeBC4_u_c12903_g1736_i12 & Sh14Contig_28260 & None \\
\hline None & Sh14Contig_58406 & None \\
\hline StHeBC4_u_c26755_g1_i1 & Sh14Contig_68980 & None \\
\hline StHeBC4_p_c26987_g0_i1 & Sh14Contig_48 & 6-4 photolyase \\
\hline StHeBC4_u_c16917_g1_i1 & Sh14Contig_50405 & None \\
\hline StHeBC4_u_c22344_g0_i6 & Sh14Contig_813 & thaumatin-like protein \\
\hline StHeBC4_u_c12903_g699_i2 & Sh14Contig_58790 & None \\
\hline StHeBC4_p_c12903_g14484_i1 & Sh14Contig_12513 & None \\
\hline StHeBC4_p_c9911_g3_i3 & Sh14Contig_54171 & predicted protein \\
\hline None & Sh14Contig_7920 & None \\
\hline StHeBC4_u_c12903_g34427_i8 & Sh14Contig_46360 & None \\
\hline
\end{tabular}




\begin{tabular}{|c|c|c|}
\hline StHeBC4_p_c12903_g35474_i1 & Sh14Contig_4481 & None \\
\hline None & Sh14Contig_43642 & None \\
\hline StHeBC4_u_c12903_g15888_i3 & Sh14Contig_81511 & None \\
\hline StHeBC4_p_c24979_g1_i1 & Sh14Contig_4792 & 97 kda heat shock protein \\
\hline StHeBC4_p_c12903_g14483_i5 & Sh14Contig_16250 & tcp transcription factor 13 \\
\hline StHeBC4_p_c12903_g6756_i 1 & Sh14Contig_12790 & None \\
\hline StHeBC4_p_c12903_g10875_i1 & Sh14Contig_20081 & beta-glucan-binding protein \\
\hline StHeBC4_p_c17648_g0_i3 & Sh14Contig_15582 & e3 ubiquitin-protein ligase upl3 \\
\hline StHeBC4_u_c12903_g22225_i1 & Sh14Contig_48959 & None \\
\hline None & Sh14Contig_45784 & None \\
\hline None & Sh14Contig_75797 & None \\
\hline StHeBC4_u_c12903_g11727_i4 & Sh14Contig_4041 & None \\
\hline StHeBC4_p_c23083_g3_i1 & Sh14Contig_37240 & None \\
\hline None & Sh14Contig_5997 & None \\
\hline None & Sh14Contig_67788 & None \\
\hline StHeBC4_p_c12903_g6756_i1 & Sh14Contig_1062 & None \\
\hline StHeBC4_u_c12903_g11833_i1 & Sh14Contig_14842 & None \\
\hline StHeBC4_p_c12903_g6700_i3 & Sh14Contig_43681 & hypothetical protein VITISV_013115 \\
\hline StHeBC4_p_c20608_g0_i7 & Sh14Contig_35726 & $\begin{array}{l}\text { PREDICTED: putative kinase-like protein } \\
\text { TMKL1-like }\end{array}$ \\
\hline None & Sh14Contig_29426 & None \\
\hline None & Sh14Contig_70066 & None \\
\hline StHeBC4_u_c12903_g29964_i1 & Sh14Contig_37086 & None \\
\hline StHeBC4_p_c12903_g39295_i4 & Sh14Contig_19126 & epoxide hydrolase 2 -like \\
\hline StHeBC4_u_c12903_g1736_i13 & Sh14Contig_29803 & None \\
\hline None & Sh14Contig_29803 & None \\
\hline StHeBC4_p_c12903_g6843_i1 & Sh14Contig_4375 & $\begin{array}{l}\text { translation initiation factor eif- } 2 b \text { subunit delta- } \\
\text { like }\end{array}$ \\
\hline StHeBC4_u_c12903_g1736_i7 & Sh14Contig_28260 & None \\
\hline StHeBC4_p_c12903_g38577_i4 & Sh14Contig_21342 & None \\
\hline StHeBC4_p_c25304_g0_i1 & Sh14Contig_5367 & serine threonine-protein kinase pbs 1 -like \\
\hline StHeBC4_u_c12903_g1736_i12 & Sh14Contig_28260 & None \\
\hline StHeBC4_p_c12903_g7992_i1 & Sh14Contig_22515 & poly-specific ribonuclease parn \\
\hline StHeBC4_u_c12903_g4208_i1 & Sh14Contig_47575 & None \\
\hline StHeBC4_p_c11471_g0_i1 & Sh14Contig_66224 & None \\
\hline StHeBC4_p_c12903_g40108_i1 & Sh14Contig_17925 & upf0326 protein at4g17486-like \\
\hline StHeBC4_p_c26825_g3_i2 & Sh14Contig_30816 & unnamed protein product \\
\hline StHeBC4_u_c17841_g0_i1 & Sh14Contig_54135 & None \\
\hline StHeBC4_h_c12903_g30936_i1 & Sh14Contig_28702 & copia LTR rider \\
\hline None & Sh14Contig_37720 & None \\
\hline StHeBC4_p_c12903_g10585_i1 & Sh14Contig_12790 & None \\
\hline StHeBC4_u_c14956_g0_i2 & Sh14Contig_3380 & None \\
\hline StHeBC4_u_c12903_g19836_i1 & Sh14Contig_44128 & None \\
\hline StHeBC4_p_c12903_g9452_i3 & Sh14Contig_68177 & None \\
\hline StHeBC4_p_c21038_g1_i1 & Sh14Contig_46012 & None \\
\hline StHeBC4_u_c12903_g35474_i11 & 1Sh14Contig_4481 & None \\
\hline StHeBC4_p_c12903_g15810_i1 & Sh14Contig_12790 & None \\
\hline StHeBC4_u_c12903_g1736_i7 & Sh14Contig_28260 & None \\
\hline StHeBC4_u_c12903_g34427_i1 & Sh14Contig_61194 & None \\
\hline
\end{tabular}




\begin{tabular}{|c|c|c|}
\hline StHeBC4_p_c18569_g2_i14 & Sh14Contig_67725 & None \\
\hline StHeBC4_u_c22214_g0_i2 & Sh14Contig_43769 & None \\
\hline StHeBC4_p_c18975_g4_i3 & Sh14Contig_77966 & hypothetical protein VITISV_037041 \\
\hline StHeBC4_p_c22472_g0_i1 & Sh14Contig_63886 & predicted protein \\
\hline StHeBC4_u_c24133_g4_i1 & Sh14Contig_30270 & None \\
\hline StHeBC4_u_c12903_g12274_i1 & Sh14Contig_5997 & None \\
\hline None & Sh14Contig_267 & None \\
\hline StHeBC4_u_c12903_g34427_i3 & Sh14Contig_31554 & None \\
\hline StHeBC4_p_c12903_g33372_i2 & Sh14Contig_18809 & serine threonine protein kinase \\
\hline StHeBC4_p_c12903_g12842_i2 & Sh14Contig_58393 & hypothetical protein VITISV_012059 \\
\hline StHeBC4_p_c12903_g13577_i4 & Sh14Contig_4336 & retrotransposon unclassified \\
\hline StHeBC4_u_c12903_g34427_i9 & Sh14Contig_46360 & None \\
\hline StHeBC4_u_c20739_g0_i7 & Sh14Contig_30629 & None \\
\hline None & Sh14Contig_64863 & None \\
\hline StHeBC4_u_c18975_g2_i1 & Sh14Contig_7369 & None \\
\hline StHeBC4_u_c3227_g1_î & Sh14Contig_65419 & None \\
\hline StHeBC4_u_c18360_g0_i1 & Sh14Contig_44903 & None \\
\hline StHeBC4_p_c12903_g6756_i1 & Sh14Contig_1062 & None \\
\hline StHeBC4_p_c12903_g20268_i2 & Sh14Contig_73193 & None \\
\hline StHeBC4_p_c12903_g15476_i8 & Sh14Contig_78156 & None \\
\hline StHeBC4_u_c12903_g29964_i1 & Sh14Contig_35346 & None \\
\hline None & Sh14Contig_62455 & None \\
\hline None & Sh14Contig_11292 & None \\
\hline StHeBC4_p_c12903_g43157_i2 & Sh14Contig_5125 & None \\
\hline None & Sh14Contig_71030 & None \\
\hline StHeBC4_u_c12903_g18073_i1 & Sh14Contig_20326 & None \\
\hline StHeBC4_p_c17480_g0_i1 & Sh14Contig_62447 & None \\
\hline StHeBC4_p_c18975_g4_i6 & Sh14Contig_44012 & F7F22.15, related \\
\hline StHeBC4_p_c12903_g32577_i4 & Sh14Contig_50541 & None \\
\hline None & Sh14Contig_78156 & None \\
\hline StHeBC4_u_c12903_g32032_i1 & Sh14Contig_20326 & None \\
\hline StHeBC4_u_c12903_g25724_i1 & Sh14Contig_32318 & None \\
\hline StHeBC4_u_c13414_g0_i1 & Sh14Contig_61194 & None \\
\hline None & Sh14Contig_43845 & None \\
\hline None & Sh14Contig_37580 & None \\
\hline StHeBC4_u_c12903_g1736_i12 & Sh14Contig_28260 & None \\
\hline StHeBC4_p_c12903_g17146_i3 & Sh14Contig_34580 & None \\
\hline StHeBC4_p_c12903_g33830_i2 & Sh14Contig_22568 & None \\
\hline StHeBC4_p_c12903_g18700_i3 & Sh14Contig_2850 & None \\
\hline None & Sh14Contig_61087 & $\begin{array}{l}\text { retrotransposon protein, putative, Ty3-gypsy } \\
\text { subclass }\end{array}$ \\
\hline StHeBC4_p_c12903_g208_i2 & Sh14Contig_7920 & None \\
\hline StHeBC4_u_c14380_g6_i22 & Sh14Contig_20089 & None \\
\hline StHeBC4_p_c12903_g22518_i1 & Sh14Contig_39552 & None \\
\hline StHeBC4_u_c18188_g0_i1 & Sh14Contig_30718 & $\begin{array}{l}\text { retrotransposon protein, putative, unclassified, } \\
\text { expressed }\end{array}$ \\
\hline StHeBC4_u_c12903_g35782_i1 & Sh14Contig_27773 & None \\
\hline None & Sh14Contig_53940 & None \\
\hline None & Sh14Contig_29803 & None \\
\hline
\end{tabular}




\begin{tabular}{|c|c|c|}
\hline \multicolumn{2}{|c|}{ StHeBC4_p_c20119_g0_i1 None } & None \\
\hline StHeBC4_p_c12903_g26306_i42 & 2None & None \\
\hline StHeBC4_u_c12903_g15888_i8 & None & None \\
\hline StHeBC4_u_c12903_g34326_i2 & None & None \\
\hline StHeBC4_u_c22214_g0_i5 & None & None \\
\hline StHeBC4_u_c12903_g32907i15 & 5 None & None \\
\hline StHeBC4_p_c15477_g0_i2 & None & None \\
\hline StHeBC4_p_c12903_g18330_i2 & None & None \\
\hline StHeBC4_u_c20631_g2_i1 & None & None \\
\hline StHeBC4_u_c18975_g1_i1 & None & None \\
\hline StHeBC4_p_c12903_g33550_i2 & None & None \\
\hline StHeBC4_u_c20348_g0_i3 & None & None \\
\hline StHeBC4_u_c19161_g0_i1 & None & None \\
\hline StHeBC4_p_c18860_g2_i3 & None & None \\
\hline StHeBC4_p_c12903_g5434_i1 & None & None \\
\hline StHeBC4_p_c12903_g34427_i2 & None & None \\
\hline StHeBC4_u_c12903_g14239_i1 & None & None \\
\hline StHeBC4_p_c12903_g16488_i2 & None & None \\
\hline StHeBC4_u_c20110_g5_i1 & None & None \\
\hline StHeBC4_u_c17278_g0_i2 & None & None \\
\hline StHeBC4_p_c18569_g0_i2 & None & None \\
\hline StHeBC4_p_c12903_g15860_i11 & 1 None & None \\
\hline StHeBC4_u_c12903_g16409_i1 & None & None \\
\hline StHeBC4_p_c12903_g5042_i2 & None & None \\
\hline StHeBC4_u_c12903_g42940_i10 & ONone & None \\
\hline StHeBC4_u_c18975_g1_i1 & None & None \\
\hline StHeBC4_u_c12903_g34427_i1 & None & None \\
\hline StHeBC4_u_c13012_g0_i1 & None & None \\
\hline StHeBC4_u_c20371_g0_i1 & None & None \\
\hline StHeBC4_u_c22472_g0_i4 & None & None \\
\hline StHeBC4_u_c12903_g34431_i3 & None & None \\
\hline StHeBC4_p_c18860_g2_i4 & None & None \\
\hline StHeBC4_p_c12903_g15230_i2 & None & None \\
\hline StHeBC4_u_c18860_g2_i48 & None & None \\
\hline StHeBC4_p_c12903_g8484_i1 & None & None \\
\hline StHeBC4_u_c18238_g1_i1 & None & None \\
\hline StHeBC4_u_c25350_g7_i2 & None & None \\
\hline StHeBC4_u_c12903_g29135_i2 & None & None \\
\hline StHeBC4_u_c13414_g0_i1 & None & None \\
\hline StHeBC4_p_c16184_g0_i1 & None & None \\
\hline StHeBC4_p_c12903_g27140_i4 & None & None \\
\hline StHeBC4_p_c12903_g18100_i3 & None & None \\
\hline StHeBC4_p_c26242_g21_i7 & None & None \\
\hline StHeBC4_u_c12903_g15860_i5 & None & None \\
\hline StHeBC4_p_c12903_g33449_i1 & None & None \\
\hline StHeBC4_u_c13012_g0_i1 & None & None \\
\hline StHeBC4_h_c10541_g0_i1 & None & None \\
\hline StHeBC4_u_c12903_g33428_i2 & None & None \\
\hline StHeBC4_u_c12903_g1552_i1 & None & None \\
\hline
\end{tabular}




$\begin{array}{lll}\text { StHeBC4_p_c16622_g1_i2 } & \text { None } & \text { None } \\ \text { StHeBC4_p_c12903_g8677_i10 } & \text { None } & \text { None } \\ \text { StHeBC4_u_c10677_g0_11 } & \text { None } & \text { None } \\ \text { StHeBC4_p_c12903_g40108_i1 } & \text { None } & \text { None } \\ \text { StHeBC4_u_c12903_g15860_i5 } & \text { None } & \text { None } \\ \text { StHeBC4_p_c19033_g0_i1 } & \text { None } & \text { None } \\ \text { StHeBC4_p_c12903_g33830_i1 } & \text { None } & \text { None } \\ \text { StHeBC4_p_c12903_g11366_i6 } & \text { None } & \text { None } \\ \text { StHeBC4_u_c12903_g25897_i6 } & \text { None } & \text { None }\end{array}$


861 Table S3. Chemocyanin variant calls for sequenced individuals. Individuals are coded as ' 1 ' if 862 they possess the 'finger millet' allele or ' 0 ' if the allele is missing.

\begin{tabular}{|c|c|c|c|c|c|c|}
\hline SampleID & Site & Host & Lat & Lon & Elevation_ft & Chemocyanin \\
\hline$\overline{\mathrm{SH} 009}$ & Mumias & maize & 0.3342 & 34.47782 & 4306 & 0 \\
\hline SH014 & Mumias & maize & 0.3342 & 34.47782 & 4306 & 0 \\
\hline SH023 & Mumias2 & maize & 0.3038 & 34.50713 & 4299 & 1 \\
\hline $\mathrm{SH} 027$ & Mumias2 & maize & 0.3038 & 34.50713 & 4299 & 0 \\
\hline SH031 & Mumias2 & maize & 0.3038 & 34.50713 & 4299 & 0 \\
\hline SH035 & Kibos & sorghum & -0.0363167 & 34.81567 & 3893 & 1 \\
\hline SH039 & Kibos & sorghum & -0.0363167 & 34.81567 & 3893 & 0 \\
\hline SH042 & Kibos & sorghum & -0.0363167 & 34.81567 & 3893 & 1 \\
\hline $\mathrm{SH} 046$ & Kibos & maize & -0.0341417 & 34.81628 & 3917 & 0 \\
\hline SH055 & Kibos & maize & -0.0341417 & 34.81628 & 3917 & 0 \\
\hline $\mathrm{SH} 065$ & Muhoroni2 & maize & -0.15125 & 35.19167 & 4239 & 0 \\
\hline SH070 & Muhoroni2 & maize & -0.15125 & 35.19167 & 4239 & 0 \\
\hline SH072 & Muhoroni2 & sugarcane & -0.15125 & 35.19167 & 4239 & 0 \\
\hline $\mathrm{SH} 074$ & Muhoroni2 & sugarcane & -0.15125 & 35.19167 & 4239 & 1 \\
\hline $\mathrm{SH} 077$ & Muhoroni2 & sugarcane & -0.15125 & 35.19167 & 4239 & 0 \\
\hline SH079 & Chemelil & maize & -0.082375 & 35.13167 & 4176 & 0 \\
\hline $\mathrm{SH} 087$ & Chemelil & maize & -0.082375 & 35.13167 & 4176 & 1 \\
\hline SH091 & Chemelil2 & sorghum & -0.0941667 & 35.12495 & 4060 & 0 \\
\hline SH097 & Chemelil2 & sorghum & -0.0941667 & 35.12495 & 4060 & 1 \\
\hline SH101 & Chemelil2 & sorghum & -0.0941667 & 35.12495 & 4060 & 0 \\
\hline SH103 & Kisii & finger millet & -0.6138 & 34.73172 & 4925 & 1 \\
\hline SH104 & Kisii & finger millet & -0.6138 & 34.73172 & 4925 & 1 \\
\hline SH105 & Kisii & finger millet & -0.6138 & 34.73172 & 4925 & 1 \\
\hline SH106 & Kisii & finger millet & -0.6138 & 34.73172 & 4925 & 1 \\
\hline SH107 & Kisii & finger millet & -0.6138 & 34.73172 & 4925 & 1 \\
\hline SH108 & Kisii & finger millet & -0.6138 & 34.73172 & 4925 & 1 \\
\hline SH109 & Kisii & finger millet & -0.6138 & 34.73172 & 4925 & 1 \\
\hline SH110 & Kisii & finger millet & -0.6138 & 34.73172 & 4925 & 1 \\
\hline $\mathrm{SH} 111$ & Kisii & finger millet & -0.6138 & 34.73172 & 4925 & 1 \\
\hline SH112 & Kisii & finger millet & -0.6138 & 34.73172 & 4925 & 0 \\
\hline SH113 & Kisii & finger millet & -0.6138 & 34.73172 & 4925 & 0 \\
\hline $\mathrm{SH} 114$ & Kisii & finger millet & -0.6138 & 34.73172 & 4925 & 0 \\
\hline SH115 & Kisii & maize & -0.6133167 & 34.7318 & 4930 & 0 \\
\hline $\mathrm{SH} 116$ & Kisii & maize & -0.6133167 & 34.7318 & 4930 & 0 \\
\hline SH117 & Kisii & maize & -0.6133167 & 34.7318 & 4930 & 0 \\
\hline SH118 & Kisii & maize & -0.6133167 & 34.7318 & 4930 & 0 \\
\hline SH119 & Kisii & maize & -0.6133167 & 34.7318 & 4930 & 0 \\
\hline SH120 & Kisii & maize & -0.6133167 & 34.7318 & 4930 & 0 \\
\hline $\mathrm{SH} 121$ & Kisii & maize & -0.6133167 & 34.7318 & 4930 & 0 \\
\hline SH122 & Kisii & maize & -0.6133167 & 34.7318 & 4930 & 0 \\
\hline $\mathrm{SH} 123$ & Kisii & maize & -0.6133167 & 34.7318 & 4930 & 0 \\
\hline $\mathrm{SH} 124$ & Kisii & maize & -0.6133167 & 34.7318 & 4930 & 0 \\
\hline SH125 & Kisii & maize & -0.6133167 & 34.7318 & 4930 & 0 \\
\hline $\mathrm{SH} 126$ & Kisii & maize & -0.6133167 & 34.7318 & 4930 & 0 \\
\hline SH127 & Homa Bay & sorghum & -0.5839667 & 34.4762 & 4173 & 0 \\
\hline
\end{tabular}




\begin{tabular}{|c|c|c|c|c|c|c|}
\hline $\mathrm{SH} 128$ & Homa Bay & sorghum & -0.5839667 & 34.4762 & 4173 & 0 \\
\hline $\mathrm{SH} 129$ & Homa Bay & sorghum & -0.5839667 & 34.4762 & 4173 & 0 \\
\hline SH130 & Homa Bay & sorghum & -0.5839667 & 34.4762 & 4173 & 0 \\
\hline $\mathrm{SH} 131$ & Homa Bay & sorghum & -0.5839667 & 34.4762 & 4173 & 0 \\
\hline SH132 & Homa Bay & sorghum & -0.5839667 & 34.4762 & 4173 & 0 \\
\hline $\mathrm{SH} 133$ & Homa Bay & sorghum & -0.5839667 & 34.4762 & 4173 & 1 \\
\hline SH134 & Homa Bay & sorghum & -0.5839667 & 34.4762 & 4173 & 0 \\
\hline $\mathrm{SH} 135$ & Homa Bay & sorghum & -0.5839667 & 34.4762 & 4173 & 0 \\
\hline $\mathrm{SH} 136$ & Homa Bay & sorghum & -0.5839667 & 34.4762 & 4173 & 0 \\
\hline $\mathrm{SH} 137$ & Homa Bay & sorghum & -0.5839667 & 34.4762 & 4173 & 0 \\
\hline $\mathrm{SH} 138$ & Homa Bay & sorghum & -0.5839667 & 34.4762 & 4173 & 0 \\
\hline SH139 & Homa Bay 2 & maize & -0.5834 & 34.47635 & 4161 & 0 \\
\hline $\mathrm{SH} 140$ & Homa Bay 2 & maize & -0.5834 & 34.47635 & 4161 & 0 \\
\hline SH141 & Homa Bay 2 & maize & -0.5834 & 34.47635 & 4161 & 0 \\
\hline $\mathrm{SH} 142$ & Homa Bay 2 & maize & -0.5834 & 34.47635 & 4161 & 1 \\
\hline $\mathrm{SH} 143$ & Homa Bay 2 & maize & -0.5834 & 34.47635 & 4161 & 1 \\
\hline SH144 & Homa Bay 2 & maize & -0.5834 & 34.47635 & 4161 & 0 \\
\hline $\mathrm{SH} 145$ & Homa Bay 2 & maize & -0.5834 & 34.47635 & 4161 & 1 \\
\hline SH146 & Homa Bay 2 & maize & -0.5834 & 34.47635 & 4161 & 0 \\
\hline $\mathrm{SH} 147$ & Homa Bay 2 & maize & -0.5834 & 34.47635 & 4161 & 0 \\
\hline $\mathrm{SH} 148$ & Homa Bay 2 & maize & -0.5834 & 34.47635 & 4161 & 0 \\
\hline SH149 & Homa Bay 2 & maize & -0.5834 & 34.47635 & 4161 & 0 \\
\hline SH150 & Homa Bay 2 & maize & -0.5834 & 34.47635 & 4161 & 0 \\
\hline
\end{tabular}



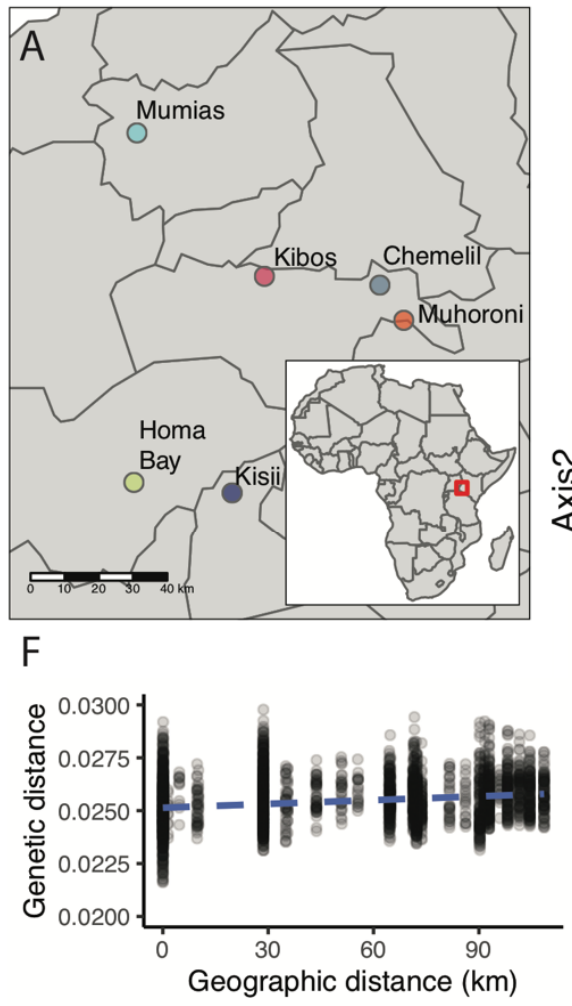

867

868

869

870

871

872

873

874

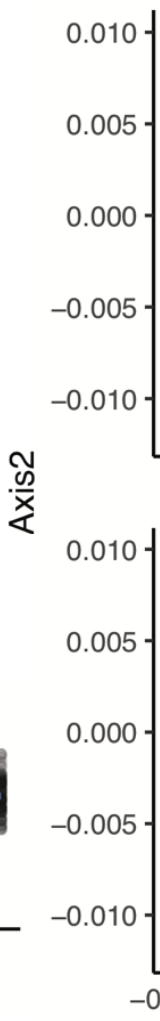

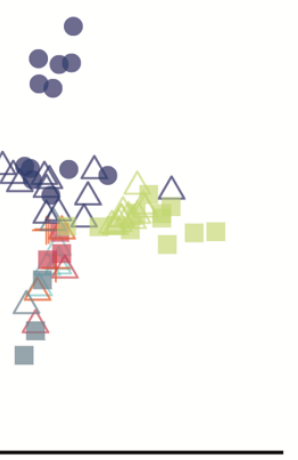

C

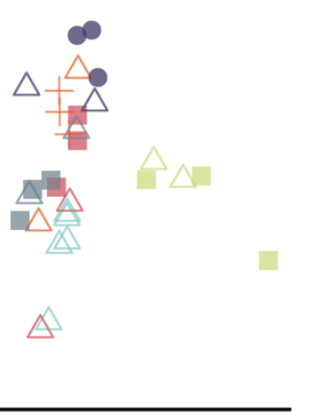

Host

- finger millet

$\triangle$ maize

- sorghum

+ sugarcane

Site

- Chemelil

Homa Bay

- Kibos

- Kisii

- Muhoroni

- Mumias

Figure 1. Population genomics of $S$. hermonthica from western Kenya. A) Map of the six sampling locations. (B-E) Principal Coordinates Analysis (PCoA) based on k-mer-derived genomic distances, performed separately for B) all sampled individuals $(n=68)$, C) five individuals per location ( $n=30)$, D) individuals from Kisii $(n=24)$, and E) individuals from Homa Bay $(n=24)$. F) Genetic vs. geographic distance. Genetic distance was based on 31-mers. The dashed blue line indicates expectations from the best fit line $\left(y=0.000013{ }^{*} \mathrm{~km}+0.0246 ; R^{2}=\right.$ 


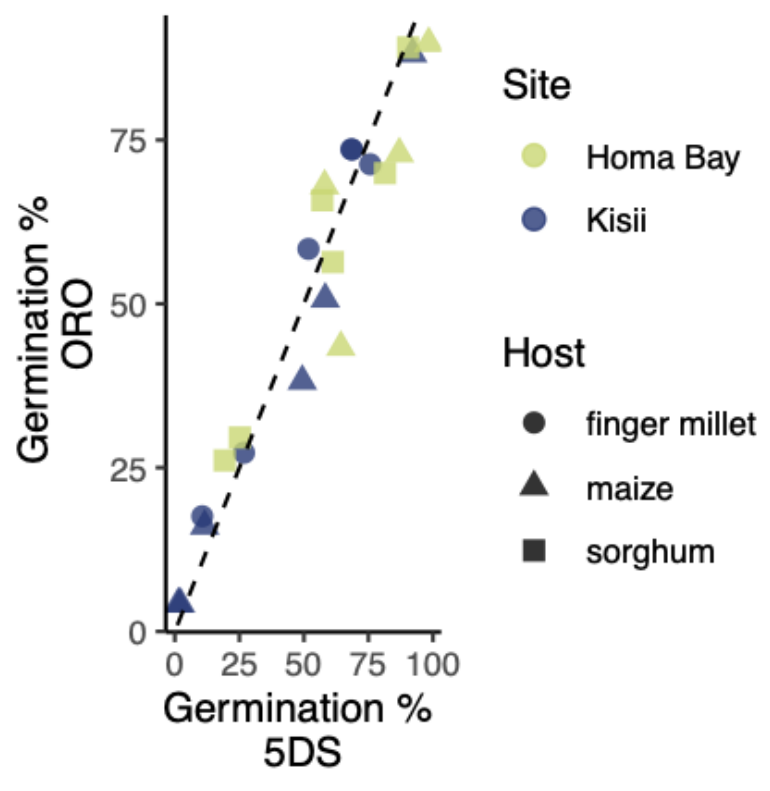

877 Figure 2. Germination response variation in Homa Bay and Kisii populations. Seeds from $n=6$

878 individuals per unique site and host were tested in response to synthetic strigolactones

879 orobanchol (ORO, $0.01 \mathrm{uM})$ and 5-deoxystrigol (5DS, $0.01 \mathrm{uM})$. The dashed line indicates the

880 expectation if the percent germination in response to the two different germination stimulants is 881 identical. 
a

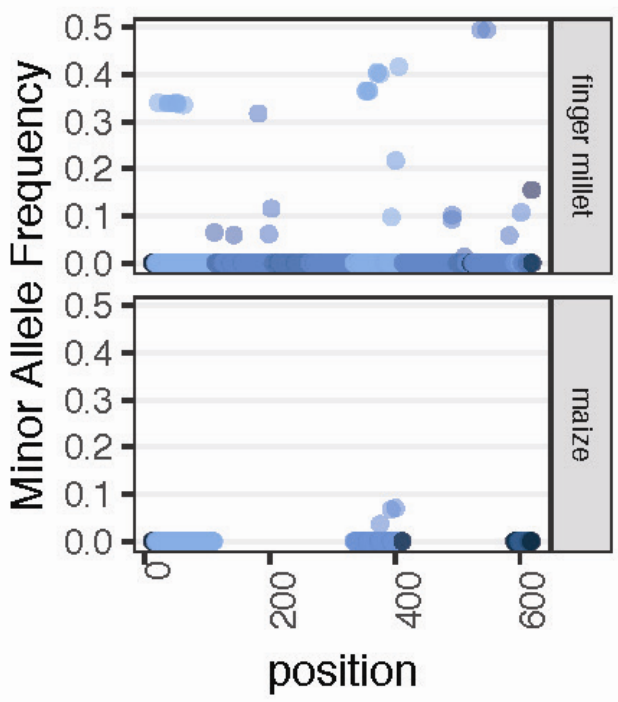

b

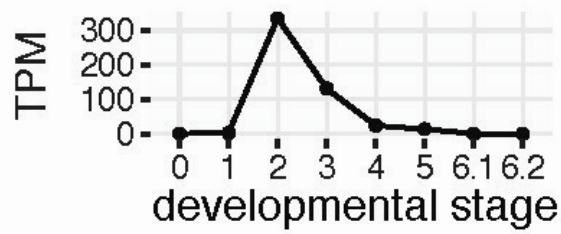

nInd

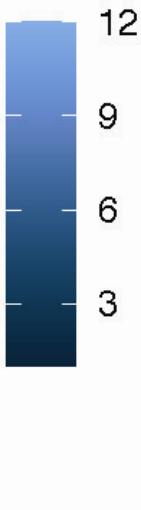

Figure 3. Genetic variation in Striga hermonthica from Kisii, Kenya. (a) Presence/absence variation across the chemocyanin precursor transcript (StHeBC4_h_c11261_g0_i1), for $n=12$ individuals per host (finger millet or maize). If the locus is not present in any of the sequenced

887 individuals, no data point is shown, otherwise each point is colored according to the number of

888 individuals ( $\mathrm{n}$ Ind) with data for the position. (b) Gene expression data in transcripts per million 889 (TPM) from the PPGPII dataset for the chemocyanin precursor transcript across six stages of 890 haustorial development (0: imbibed seed; 1 : germinated seedling after exposure to GR24; 2 :

891 germinated seedling after exposure to DMBQ; 3: 48 hrs post-attachment; 4: 72 hrs post-

892 attachment; 5: late post-attachment; 6.1: vegetative structures; 6.2: reproductive structures). (c) 893 Distribution of Tajima's D and $F_{S T}$ values across 154,722 non-overlapping 1-kb windows. Only 894 windows with data for more than $50 \%$ of sites are shown, excluding impacts of structural genetic 895 variation as in (a). Tajima's D for the chemocyanin was -1.7. 